\title{
The role of antimiR-26a-5p/biphasic calcium phosphate in repairing rat femoral defects
}

\author{
XIAOYAN YUAN ${ }^{1,2^{*}}$, LU HAN $^{1,3^{*}}$, HAI LIN $^{4 *}$, ZEYOU GUO $^{1,3}$, YANLING HUANG $^{1}$,

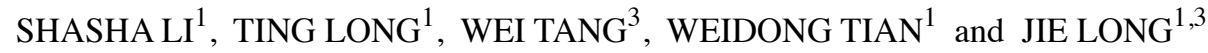

\author{
${ }^{1}$ The State Key Laboratory of Oral Diseases, Sichuan University, Chengdu, Sichuan 610041; ${ }^{2}$ Department of \\ Aesthetic Medicine, The Second People's Hospital of Chengdu, Chengdu, Sichuan 610017; ${ }^{3}$ Department of Oral and \\ Maxillofacial Surgery, West China College of Stomatology, Sichuan University, Chengdu, Sichuan 610041; \\ ${ }^{4}$ National Engineering Research Center for Biomaterials, Sichuan University, Chengdu, Sichuan 610064, P.R. China
}

Received October 30, 2018; Accepted June 19, 2019

DOI: $10.3892 /$ ijmm.2019.4249

\begin{abstract}
Although miRNAs have been implicated in the osteogenic differentiation of stem cells, their role in bone repair and reconstruction in tissue-engineered bone grafts remains unclear. We previously reported that microRNA (miR)-26a-5p inhibited the osteogenic differentiation of adipose-derived mesenchymal stem cells (ADSCs), and that antimiR-26a-5p exerted the opposite effect. In the present study, the role of miR-26a-5p- and antimiR-26a-5p-modified ADSCs combined with biphasic calcium phosphate (BCP) scaffolds was evaluated in a rat femur defect model. The aim of the present study was to improve the understanding of the role of miR-26a-5p in bone regeneration in vivo, as well as to provide a new method to optimize the osteogenic ability of BCPs. ADSCs were infected with Lv-miR-26a-5p, Lv-miR-NC, Lv-antimiR-26a-5p or Lv-antimiR-NC respectively, and then combined with BCP scaffolds to repair rat femoral defects. Using X-rays, micro-computed tomography and histology at 2,4 , and 8 weeks postoperatively, the quantity and rate of bone regeneration were analyzed, revealing that they were the highest in animals treated with antimiR-26a-5p and the lowest in the miR-26a-5p treatment group. The expression levels of osteocalcin, collagen I, Runt-related transcription factor 2 , Wnt family member $5 \mathrm{~A}$ and calmodulin-dependent protein kinase II proteins were positively correlated with the bone formation rate. Taken together, the present results demonstrated that miR-26a-5p
\end{abstract}

Correspondence to: Professor Jie Long, The State Key Laboratory of Oral Diseases, Sichuan University, 14, The 3rd Section of South People's Road, Chengdu, Sichuan 610041, P.R. China

E-mail: dr.jielong@hotmail.com

*Contributed equally

Key words: anti-microRNA-26a-5p, adipose-derived mesenchymal stem cells, biphasic calcium phosphate, $\mathrm{Wnt} / \mathrm{Ca}^{2+}$ pathway, femoral bone regeneration inhibited bone formation while antimiR-26a-5p accelerated bone formation via the $\mathrm{Wnt} / \mathrm{Ca}^{2+}$ signaling pathway. Therefore, antimiR-26a-5p-modified ADSCs combined with BCP scaffolds may be used to construct an effective tissue-engineering bone graft for bone repair and reconstruction.

\section{Introduction}

Reconstruction of bone defects, especially massive segmental defects, remains a major challenge. Various approaches have been reported as suitable reconstruction techniques, but all of them are associated with different disadvantages, which limit their wide application $(1,2)$. Although autografts and allografts usually result in satisfactory outcomes, they also have many shortcomings, including, wound infection, bone graft exposure and graft failure (3-5). There has been a recent focus on tissue-engineered bone grafts (TEBGs) as an alternative to autografts for bone repair and reconstruction $(6,7)$. Autografts generally result in satisfactory healing outcomes because of their similar composition to the bone matrix as a scaffold for bone regeneration, as well as the presence of living differentiated and progenitor cells, leading to osteointegration and osteoconduction (8). Notably, the activity of the growth factors present in autografts has been shown to mediate new bone formation through signaling and the recruitment of osteoprogenitor cells (9). The three major components of TEBGs include scaffolds, cells and cell factors that promote osteogenesis (10). TEBGs are constructed based on the composition of autografts. Scaffolds are made of biomaterials, which have the advantages of unlimited supplies, safety, biocompatibility, and bioactivity; they are also capable of inducing the differentiation of stem cells (11). Biphasic calcium phosphates (BCPs), which are a combination of hydroxyapatite (HA) and $\beta$-tricalcium phosphate ( $\beta$-TCP), have been shown to be able to achieve a good outcome in repairing small defects in the clinic (12). However, they cannot be used for load-bearing areas or massive segmental defects, especially critical-size defects, since it is difficult to find an optimal ratio of HA/ $\beta$-TCP to balance the degradation of the scaffold and the rate of bone formation, which guarantees the stability of the tissue-engineered bone while promoting bone 
formation (13). Overcoming rapid scaffold degradation therefore involves improving the quantity, quality and rate of new bone formation during the treatment of critically-sized skeletal defects by BCPs.

It was previously reported that the addition of autologic osteoblasts to biomaterials promoted new bone formation, and increased levels of growth factors such as bone morphogenetic protein 2 (BMP-2) (14,15). However, the short-lived in vivo efficacy of BMP-2 and the difficulty of osteoblast isolation limits their usage $(16,17)$. Recent studies have therefore focused on the combination of osteogenic gene-modified mesenchymal stem cells (MSCs) and BCPs for TEBGs, and an important goal is to identify the most effective osteogenic genes in this process $(18,19)$.

Osteoblast differentiation is a fundamental step during bone formation, and is regulated via several signaling pathways including transforming growth factor- $\beta, \mathrm{BMP}$, and WNT, as well as a number of transcription factors that are regulated by microRNAs (miRNAs/miRs) (20-23). Understanding the signaling pathways and regulators of osteogenesis is critical for also understanding the mechanism underlying bone formation, and for developing novel strategies for bone repair and reconstruction.

miRNAs are endogenous, eukaryotic highly conserved, noncoding RNAs of 19-25 nucleotides in length, which function by complementary pairing to the 3 -untranslated region (UTR) of target mRNAs to inhibit their translation (24). Some miRNAs were recently shown to be able to regulate the osteogenic differentiation of MSCs in vitro; these included miRNA-154-5p, miRNA-30, miRNA-29b, miRNA-133 and miRNA-135 (24-28). However, it is not clear if miRNAs play a role in osteogenesis in vivo. We recently showed that downregulating endogenous miRNA-26a-5p (antimiR-26a-5p) promoted the osteogenic differentiation of ADSCs (29). These data suggested that antimiR-26a-5p-modified ADSCs combined with BCP scaffolds may accelerate new bone formation and compensate for the rapid degradation of this biomaterial. However, this hypothesis has not been verified.

The present study analyzed the effects of miR-26a-5p on bone formation by transplanting rat femoral defect models with TEBGs constructed usingmiR-26a-5p- and antimiR-26a-5p-modified ADSCs combined with BCP scaffolds. The molecular mechanism of this phenomenon was also investigated by evaluating the expression of key proteins in the $\mathrm{Wnt} / \mathrm{Ca}^{2+}$ signaling pathway that were implicated in our previous study (29). The aim of the present study was to improve the understanding of the role of miR-26a-5p in bone regeneration, to verify whether the results in vitro obtained by our previous work corresponded with those of the present experiments in vivo, and to potentially provide a new method to optimize the osteogenic ability of BCPs.

\section{Materials and methods}

Isolation, culture and identification of ADSCs. A total of 10 four-week-old female Sprague-Dawley (SD) rats (weight, $75 \mathrm{~g}$ ) obtained from the Sichuan University Animal Experimental Center were used as the source of ADSCs, and all procedures were approved by the Animal Research Committee of Sichuan University. Animals were maintained in the same room under standard conditions including temperature $\left(22-24^{\circ} \mathrm{C}\right)$, humidity $(55-65 \%)$ and light (12-h light/dark cycle) with free access to food and tap water. The ADSCs were harvested from the inguinal fat pads of the rats as previously described (30). Briefly, the excised inguinal fat pads were soaked in PBS with $1 \%$ penicillin/streptomycin, and washed 3 times with PBS. They were then minced into small pieces, and digested with $0.2 \%$ collagenase type $\mathrm{I}$ at $37^{\circ} \mathrm{C}$ in a shaking incubator for $45 \mathrm{~min}$. After centrifugation at $200 \mathrm{x} \mathrm{g}$ for $5 \mathrm{~min}$ at room temperature, the cells were resuspended in $3 \mathrm{ml}$ regular growth medium consisting of Minimum Essential Medium $\alpha$-medium ( $\alpha$-MEM; HyClone; GE Healthcare Life Sciences), $10 \%$ fetal bovine serum (Gibco; Thermo Fisher Scientific, Inc.), penicillin (100 U/ml)/streptomycin $(100 \mu \mathrm{g} / \mathrm{ml}$; Sigma-Aldrich; Merck KGaA), and cultured in T25 culture flasks (BD Falcon Labware; BD Biosciences) at $37^{\circ} \mathrm{C}$ in the presence of $5 \% \mathrm{CO}_{2}$ and $95 \%$ humidity. The culture medium was renewed every 2 days. Upon reaching $80 \%$ confluence, the adherent cells were resuspended with $0.25 \%$ trypsin and subcultured. After 2 weeks of culture, the multipotent differentiation of ADSCs (passage 3) was demonstrated based on alizarin red staining, oil red-O staining and $\beta$ III-tubulin immunofluorescence staining of transdifferentiated cells as described previously $(25,31)$ (Fig. 1A-D). Cells from passage 3 were used for all subsequent experiments.

Preparation of lentivirus vectors and infection. The construction of the lentiviral vectors containing miR-26a-5p, miR-negative control (NC), antimiR-26a-5p and antimiR-NC as well as the sequences were described in our previous study (29). ADSCs were seeded in 6-well plates $\left(4 \times 10^{5}\right.$ cells/well) one day before infection. Cells were then incubated overnight with Lv-miR-26a-5p, Lv-miR-NC, Lv-antimiR-26a-5p or Lv-antimiR-NC at final concentrations of $100 \mathrm{nM}$ with Lipofectamine 2000 ${ }^{\mathrm{TM}}$ (Invitrogen; Thermo Fisher Scientific, Inc.) in Opti-MEM ${ }^{\circledR}$ (Gibco; Thermo Fisher Scientific, Inc.), according to the manufacturer's protocol. At $72 \mathrm{~h}$ post-infection, the expression of green fluorescent protein was measured under fluorescence microscopy to evaluate the transfection efficiency. The transfection efficiency was $>95 \%$ in all groups (Fig. 1E and F). The transfection efficiency of Lv-miR-26a-5p, Lv-miR-NC, Lv-antimiR-26a-5p or Lv-antimiR-NC was verified by reverse transcription-quantitative PCR as described in our previous study (29) (Fig. 1J).

Preparation of BCP scaffolds and cell seeding. All of the BCP scaffolds, shaped into $4 \times 4 \times 2 \mathrm{~mm}^{3}$ squares, were obtained from the National Engineering Research Center for Biomaterials of Sichuan University, and sterilized by $\gamma$-irradiation at $25 \mathrm{kGy}$. The BCP20/80 used in the present study was composed of $20 \% \mathrm{HA}$ and $80 \% \beta$-TCP. The porosity was $\sim 74 \%$, and the diameter of the pores was $100-400 \mu \mathrm{m}$.

All of the BCP scaffolds were divided randomly and equally into five groups (miR, miR-NC, antimiR, antimiR-NC and Control groups) and were conditioned with $\alpha$-MEM one day before cell seeding. A total of 9BCP scaffolds from each group were used for cell adhesion evaluation and cell proliferation assays, and the remaining 18 scaffolds from each group were used in the animal experiments. ADSCs infected with Lv-miR-26a-5p, Lv-miR-NC, Lv-antimiR-26a-5p, or 

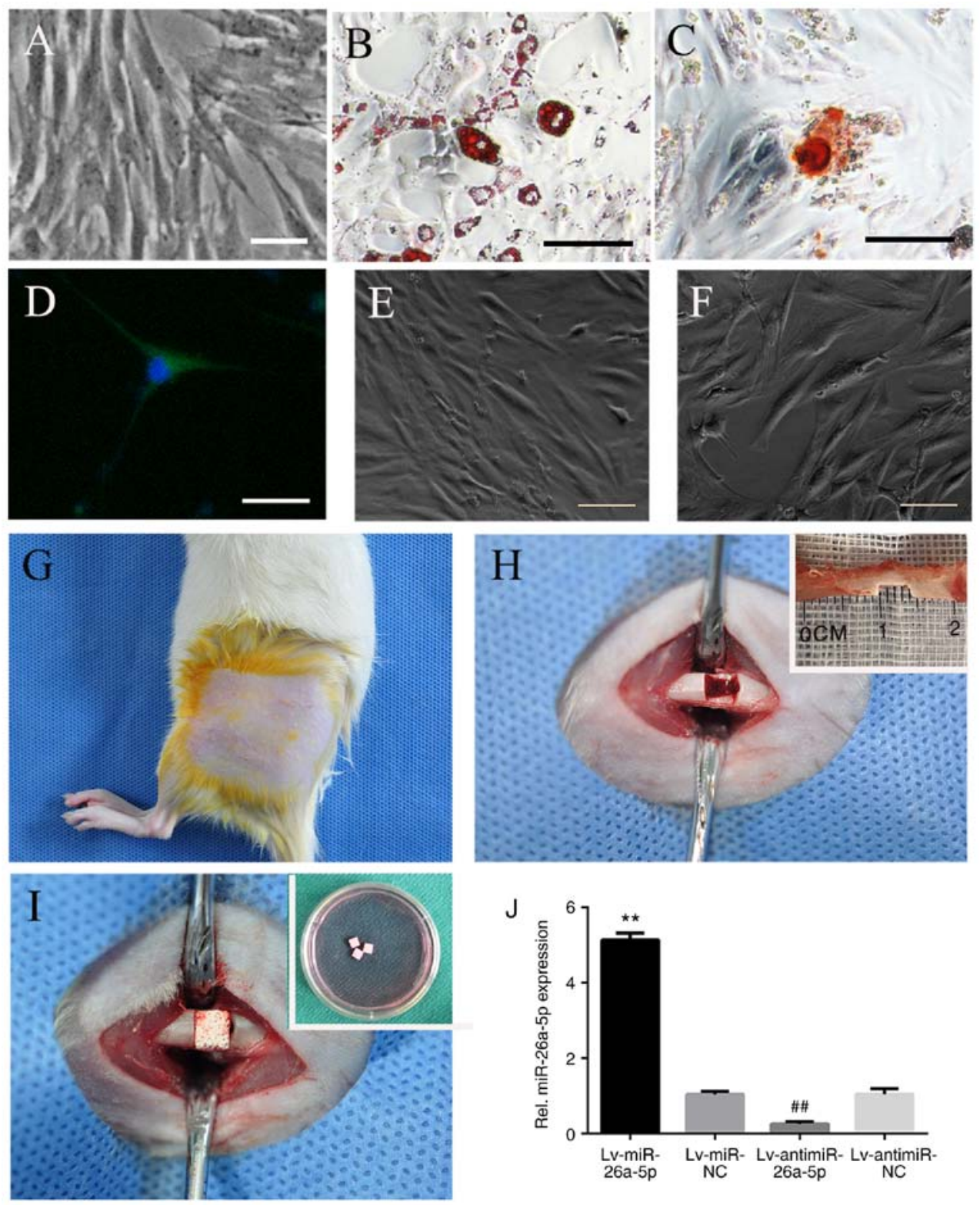

Figure 1. Identification of ADSCs and surgical procedures. (A) The fusiform shape and adherent growth of ADSCs (passage 3), (B) Oil red-O staining of adipogenic differentiated ADSCs, (C) Alizarin red staining of osteogenic differentiated ADSCs, and (D) BIII-tubulin immunofluorescence identification of neurogenic differentiated ADSCs. Identification of lentivirus infection: (E) Morphology of ADSCs before infection and (F) transfection efficiency of lentivirus was estimated based on green fluorescent protein expression in ADSCs observed through fluorescence microscopy. Scale bars, $50 \mu \mathrm{m}$. Surgical procedures: (G) Left thigh incision in Sprague-Dawley rats; (H) exposure of left femoral diaphysis, and $4 \mathrm{~mm}$ long defect made on the medial third of diaphysis; and (I) implantation of cell-scaffold complex (biphasic calcium phosphate scaffold and ADSCs) into the femoral defect. (J) Expression of miR-26a-5p in rat ADSCs infected with Lv-miR-26a-5p, Lv-miR-NC, Lv-antimiR-26a-5p and Lv-antimiR-NC were assayed by reverse transcription-quantitative PCR at $72 \mathrm{~h}$ after induction. ${ }^{* *} \mathrm{P}<0.01$ vs. miR-NC; ${ }^{\# /} \mathrm{P}<0.01$ vs. antimiR-NC. miR, microRNA; NC, negative control; ADSCs, adipose-derived mesenchymal stem cells; Lv, lentivirus.

Lv-antimiR-NC, and ADSCs (passage 3) were harvested with $0.25 \%$ trypsin (at $37^{\circ} \mathrm{C}$ for $1 \mathrm{~min}$ ), counted with a hemocytometer, and were loaded onto 5 the corresponding groups of $\mathrm{BCP}$ scaffolds $\left(1 \times 10^{5}\right.$ cells/scaffold). Cells were cultured in regular growth medium overnight for cell adhesion. The miR group consisted of miR-26a-5p-modified ADSCs/BCP; the miR-NC group consisted of ADSCs infected with Lv-miR-NC/BCP (the negative control of the miR group); the antimiR group consisted ofantimiR-26a-5p-modified ADSCs/BCP; the antimiR-NC group consisted of ADSCs infected with Lv-antimiR-NC/BCP (the negative control of the antimiR group); and the Control group consisted of ADSCs/BCP (the empty control).
Evaluation of cell adhesion and proliferation. After 7 days of incubation, 3 cell-scaffold structures from each group were fixed separately in $4 \%$ paraformaldehyde solution for 24 hat room temperature, followed by dehydration with an ethanol concentration series of $30-100 \%$ and dried in a dryer. One scaffold without cells was cultured and subjected to the same protocol to be used as the blank control. The structures were then sputter coated with gold and subjected to scanning electron microscopy (SEM; FEI Quanta 200 ESEM; Thermo Fisher Scientific, Inc.) to evaluate cell adhesion.

On days 1 and 7 of incubation, a ADSC cell proliferation assay foreach group was performed using Cell Counting Kit-8 (CCK8; Dojindo Molecular Technologies, Inc.). Briefly, 
CCK- 8 solution was added to the medium (CCK-8:medium, 1:10). After incubation for 2 hat $37^{\circ} \mathrm{C}$, the supernatant was transferred into a 96 well plate and the absorbance was read at $450 \mathrm{~nm}$ with a microplate reader (BioTek Instruments, Inc.).

Animals and surgical procedures. A total of 90 adult female SD rats (300 g; 10-weeks-old) supplied by the Sichuan University Animal Experimental Center were used to establish the femoral defect model in order to study the efficiency of bone regeneration. Animals were maintained under standard conditions including temperature $\left(22-24^{\circ} \mathrm{C}\right)$, humidity $(55-65 \%)$ and light (12-h light/dark cycle) with free access to food and tap water. The surgical procedures and experimental protocols for animal care were approved by the Animal Research Committee of Sichuan University. The SD rats were randomly divided into 5 groups (miR, miR-NC, antimiR, antimiR-NC and Control groups), with 18 rats in each group, corresponding to the 5 groups of cell-scaffold structures (Table I).

Surgery was performed under aseptic conditions. Prior to surgery, the rats were anesthetized with an intraperitoneal injection of $10 \%$ chloral hydrate $(300 \mathrm{mg} / \mathrm{kg})$, the left thigh was shaved, and the animal received a subcutaneous injection of $2 \%$ lidocaine $(4 \mathrm{mg} / \mathrm{kg}$ ) for local anesthesia (Fig. $1 \mathrm{G})$. The left femur was exposed by a lateral longitudinal skin incision and by retracting the quadriceps muscles. The incision was $2.5 \mathrm{~cm}$ long in order to expose the medial part of the femoral diaphysis. After the periosteum was stripped off the bone, a $4 \mathrm{~mm}$-long $\mathrm{x} 2 \mathrm{~mm}$-deep defect was made by a pneumatic fissure bur under sustained saline irrigation (Fig. 1H). Then the cell-scaffold structure was transplanted into the defect (Fig. 1I). Subsequently, the incision was closed by suturing. After surgery, the experimental rat received a penicillin injection (20 U/day) once a day for 3 days and the wound was cleaned every day for a week to prevent infection. If there was any sign of local infection, the anti-infection and wound-cleaning treatment was prolonged.

At 2, 4 or 8 weeks postoperatively, 6 animals from each group were sacrificed randomly by cervical dislocation after anesthesia to obtain left femur samples. Prior to euthanasia, the rats were administered an intraperitoneal injection of $10 \%$ chloral hydrate $(300 \mathrm{mg} / \mathrm{kg}$ ) for general anesthesia. Cervical dislocation was then conducted by an experienced researcher very quickly and no rats showed clinical signs of suffering before they died. Only after the lack of a heartbeat had been confirmed and their bodies became cold, were samples obtained according to laboratory standards. In the present study, several signs of suffering were considered to indicate the requirement of humane endpoints such as severe infections, uncontrolled body weight loss or apastia over $36 \mathrm{~h}$ and bone fracture.

$X$-ray analysis. The periosteal tissue was stripped out of the femur diaphysis. Each sample was placed on an occlusal film, then a standardized anteroposterior radiograph of the femur was performed with an X-ray unit (CS 2100; Carestream Dental LLC). The exposure conditions were $70 \mathrm{kV}, 8 \mathrm{~mA}$ and $0.06 \mathrm{msec}$.

Micro-computed tomography (CT) analysis. After X-ray films were taken, all of the samples were fixed in $4 \%$ paraformaldehyde at room temperature. After $24 \mathrm{~h}$, the samples were
Table I. Subject groups and study design.

\begin{tabular}{lcl}
\hline Groups & No. of rats & \multicolumn{1}{c}{ Treatment } \\
\hline miR & 18 & miR-26a-5p/ADSCs/BCP \\
miR-NC & 18 & miR-NC/ADSCs/BCP \\
AntimiR & 18 & AntimiR-26a-5p/ADSCs/BCP \\
AntimiR-NC & 18 & AntimiR-NC/ADSCs/BCP \\
Control & 18 & ADSCs/BCP \\
\hline
\end{tabular}

miR, microRNA; NC, negative control; ADSCs, adipose-derived mesenchymal stem cells; BCP, biphasic calcium phosphate.

dissected to keep the regenerated bone and $4 \mathrm{~mm}$ of the native bone bilaterally beneath the defect, and then samples were placed in mid-sized sample tubes with $4 \%$ paraformaldehyde. The scans were performed in an axial direction parallel to the long axis of the samples with a micro-CT machine ( $\mu \mathrm{CT}-50$; SCANCO Medical) with medium-resolution (500 projections $/ 180^{\circ}$ ) and a voxel size of $10 \mu \mathrm{m}$. The system was set to $70 \mathrm{kV}, 114 \mathrm{~mA}$, and $400 \mathrm{msec}$ integration time. The sagittal images of each sample were captured and regions of interest (ROIs) were outlined as regenerated bones, including 300 slices. The threshold of each image was set as 220-520 to isolate new bone from residual BCP. Within the ROI, the micro-architecture parameters of the new bone were evaluated, including the ratio of bone volume to total volume (BV/TV), bone mineral density (BMD), trabecular number (Tb.N) and trabecular thickness (Tb. Th) using the recommended software (NRecon 1.6 and CTAn 1.8; Bruker Corporation). The ratio of the residual BCP volume to the TV of the ROI was also evaluated using the software.

Histological analysis. All samples were fixed in $4 \%$ paraformaldehyde for 72 hat room temperature, decalcified in $20 \%$ ethylenediaminetetraacetic acid (EDTA) for 8 weeks, and then dehydrated with a concentration series of alcohol. Samples were embedded in paraffin and sectioned along the longitudinal axis of the samples into $5 \mu \mathrm{m}$ thick slices. Two sections of each sample were stained with hematoxylin and eosin (H\&E) at room temperature for $11 \mathrm{~min}$. DP2-BSW version 2.1 software (Olympus Corporation) coupled with a light microscope were used to capture the images of each section. The relative volume of newly formed bone in the defect area was measured with ImageJ 1.49p software (National Institute of Health).

Immunohistochemical (IHC) analysis. IHC staining was performed to detect the expression of osteocalcin (OCN), collagen I (COLI), Runt-related transcription factor 2(RUNX2), Wnt family member 5A (WNT5A) and calmodulin-dependent protein kinase II (CaMKII) proteins in the same $5 \mu \mathrm{m}$-thick sections used for H\&E staining. The sections were firstly deparaffinized, and then incubated with $3 \%$ hydrogen peroxide and distilled water at room temperature for $10-20 \mathrm{~min}$ to eliminate endogenous peroxidase. Samples were washed three times with double-distilled water, treated with $10 \mathrm{mM}$ sodium citrate ( $\mathrm{pH}$ 6.0) at a high pressure (room temperature) for 3 min for antigen retrieval and then washed with 0.01 M PBS (pH 7.4) after allowing to naturally cool. Antigen blocking 


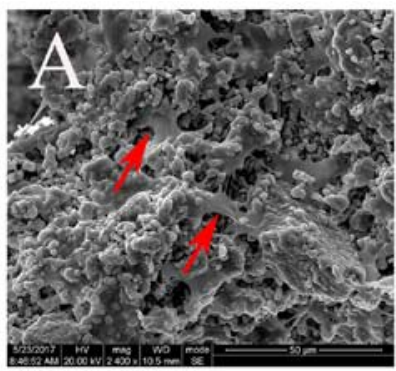

miR-26a-5p/ADSCs/BCP

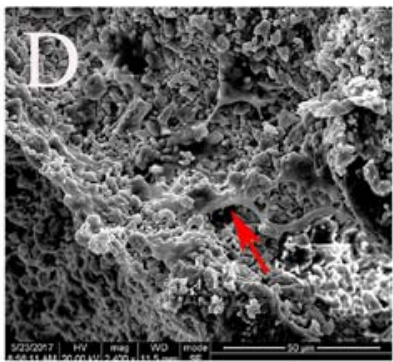

antimiR-NC/ADSCs/BCP

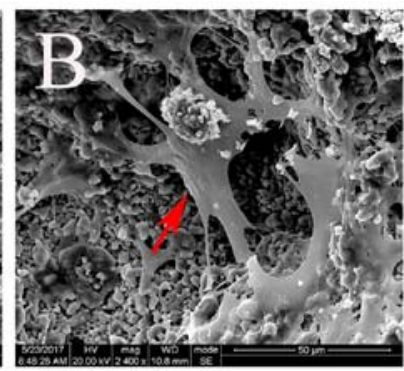

miR-NC/ADSCs/BCP

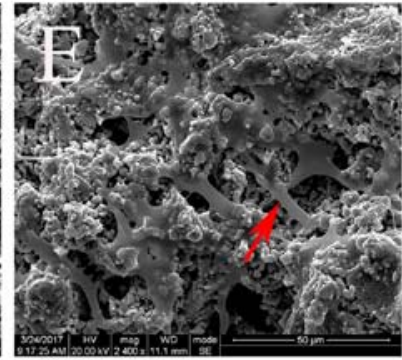

ADSCs/BCP

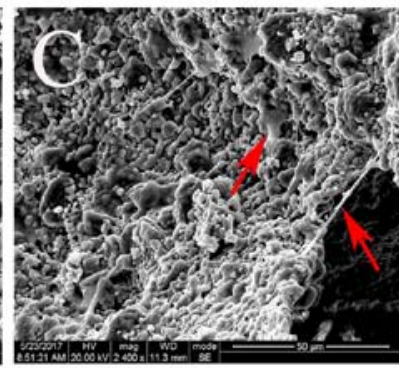

antimiR-26a-5p/ADSCs/BCP

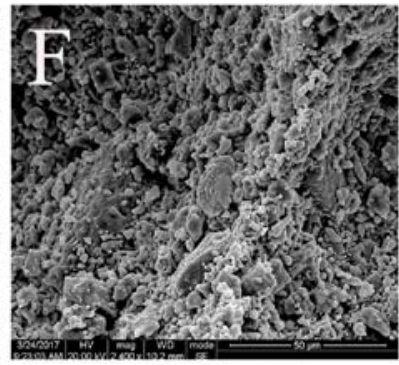

BCP scaffold only

$\mathrm{G}$

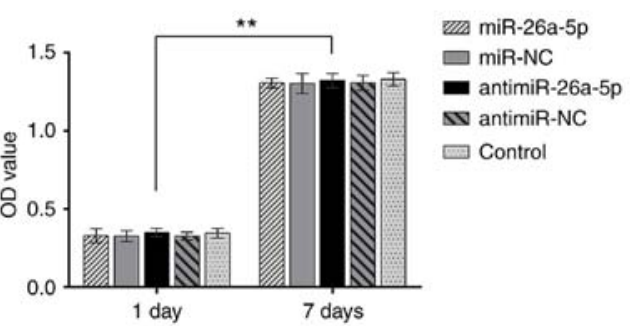

Figure 2. Scanning electron microscopy evaluation of ADSCs seeded on the BCP scaffolds. (A) ADSCs infected with Lv-miR-26a-5p (miR group); (B) ADSCs infected with Lv-miR-NC (miR-NC group); (C) ADSCs infected with Lv-antimiR-26a-5p (antimiR group); (D) ADSCs infected with Lv-antimiR-NC (antimiR-NC group); and (E) unmodified ADSCs tightly adhered to the BCP scaffolds (Control group). (F) No cells on the BCP scaffold. The red arrows indicate cells on the scaffolds. Scale bars, $50 \mu \mathrm{m}$. (G) The cell proliferation of ADSCs on the BCP scaffolds was examined by Cell Counting Kit- 8 assay. ${ }^{* *} \mathrm{P}<0.01$, as indicated. miR, microRNA; NC, negative control; ADSCs, adipose-derived mesenchymal stem cells; Lv, lentivirus; BCP, biphasic calcium phosphate; d, days; OD, optical density.

was conducted via the dropwise addition of 5-10\% normal goat serum (ZSGB-BIO; OriGene Technologies, Inc.) to the sections at $37^{\circ} \mathrm{C}$ for $10-30 \mathrm{~min}$. The serum was then removed, and samples were incubated overnight at $4^{\circ} \mathrm{C}$ with the appropriate dilutions of primary antibodies specific to the following biomarkers: OCN (cat. no. ab13418; 1:400; Abcam), COLI (cat. no. ab34710; 1:200; Abcam), RUNX2 (cat. no. ab76956; 1:200; Abcam), WNT5A (cat. no. Gxp536895-50 ug; 1:200; GenXspan) and CaMKII (cat. no. Gxp63948-50 ug; 1:200; GenXspan). The sections were then washed three times with PBS, and biotinylated goat anti-rabbit secondary antibodies (cat. no. sp9001; 1:400; ZSGB-BIO; OriGene Technologies, Inc.) were added dropwise on COLI, WNT5A and CaMKII sections, and incubated for 10-30 min, while biotinylated goat anti-rat secondary antibodies (cat. no. sp9002; 1:400; ZSGB-BIO; OriGene Technologies, Inc.) were added dropwise on OCN and RUNX2 sections and incubated for 10-30 min, all at room temperature. Samples were washed, and incubated with streptavidin-horseradish peroxidase conjugate followed by 3,3'-diaminobenzidine treatment. Finally, the nuclei were counterstained with hematoxylin at room temperature for $1 \mathrm{~min}$ before the sections were dehydrated and placed on coverslips. The sections for blank control were incubated with PBS instead of primary antibody. DP2-BSW version 2.1 Software (Olympus Corporation) coupled with a light microscope were used to capture the images of regenerated bone in each section (magnification, $\mathrm{x} 400$ ). Osteoblasts positive for OCN, COLI, RUNX2, WNT5A and CaMKII expression were stained yellow or brown and assessed by ImageJ 1.49 p software (National Institutes of Health). The mean area of positive cells was measured and used as the evaluation index.

Statistical analysis. All experiments were performed at least three times. Data were expressed as the mean \pm standard derivation. The data obtained were analyzed using SPSS 20.0 (IBM Corp.). All comparisons were performed using Student's two-tailed t-test or a one-way ANOVA. The Student-Newman-Keuls-q test was used as the post hoc test for multiple comparisons. Statistical values were illustrated using GraphPad Prism, version 6.05 (GraphPad Software, Inc.). $\mathrm{P}<0.05$ was considered to indicate a statistically significant difference.

\section{Results}

SEM evaluation of cell adhesion and CCK-8 assay evaluation of cell proliferation. The SEM images revealed that the ADSCs successfully and tightly adhered to the BCP scaffolds. There was an adequate number of cells on each of the scaffolds in all five groups (Fig. 2A-F). The ADSCs on the scaffolds had a fusiform or polygon morphology. 


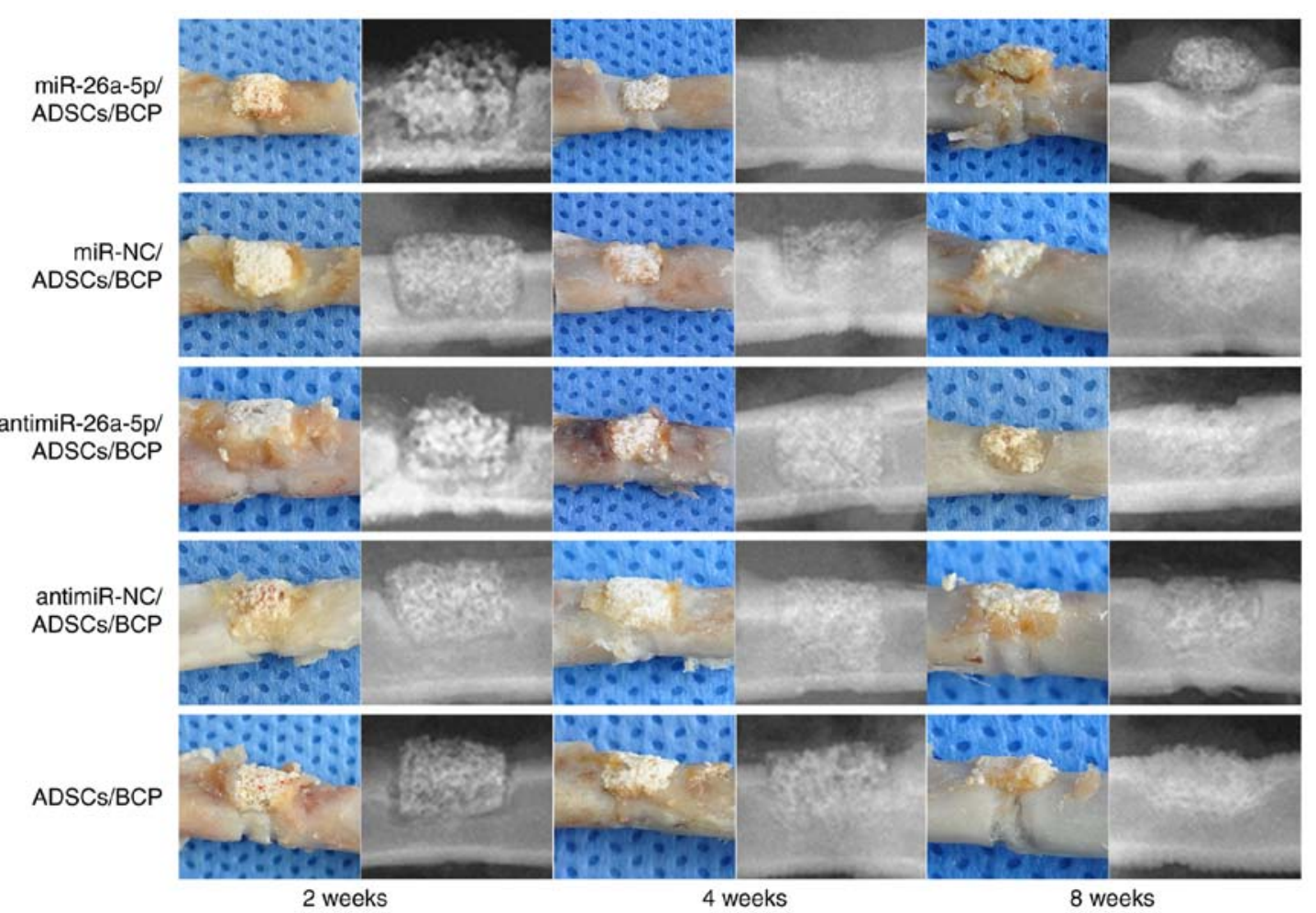

Figure 3. Gross observation and radiographical analysis of left rat femoral defects repaired by tissue-engineered bone grafts in the miR, miR-NC, antimiR, antimiR-NC and Control groups at 2,4 and 8 weeks, postoperatively. Significant new bone formation and BCP degradation were observed with the disappearance of the osteotomy line and diminished area of defects at 8 weeks postoperatively. miR, microRNA; NC, negative control; ADSCs, adipose-derived mesenchymal stem cells; BCP, biphasic calcium phosphate.

The proliferation of ADSCs on the BCP scaffolds of each group was confirmed by CCK-8 assay. Following 7 days of incubation, the number of ADSCs on the BCP scaffolds was significantly increased compared with 1 day of incubation $(\mathrm{P}<0.01)$; however, no statistically significant differences between the five groups at the same time point were identified (Fig. 2G).

Gross observation and radiographical analysis of bone regeneration. All experimental animals were healthy and successfully completed the experiment. No rats presented any signs of peritonitis following the administration of $10 \%$ chloral hydrate during the study. The maximum percentage of body weight loss observed in this study was $\sim 8 \%$.

At 2,4 and 8 weeks postoperatively, 6 animals from each group were chosen randomly and sacrificed by euthanasia. Left femur samples were harvested for gross observation and X-ray testing (Fig. 3). All 90 experimental samples were obtained from 90 different rats (30 rats were sacrificed at each time point; $n=6$ rats from each group per time point). The BCP scaffolds were stably connected to the native bones, and there was some connective tissue surrounding the scaffolds. Radiographical analysis revealed that although the lines between BCP and native bones could be clearly identified at 2 weeks postoperatively, they became indistinct at 4 weeks postoperatively as the $\mathrm{BCP}$ was beginning to be absorbed. At 8 weeks postoperatively, there was evident new bone formation in the defects, with the BCP scaffolds becoming smaller when compared with at 4 weeks. From the radiographical analyses, the boundaries between $\mathrm{BCP}$ and native bones were shown to disappear and the BCP scaffolds were greatly diminished and fused with the regenerative bones except the miR group. The residual $\mathrm{BCP}$ did not connect tightly with the newly formed bone in the miR group.

Micro-CT analysis of bone regeneration. At 2,4 and 8 weeks postoperatively, new bone formation in the ROI was measured by micro-CT (Fig. 4). Regenerated bone in the defect increased over time in all 5 groups, starting from surrounding the BCP scaffolds, gradually filling the macro-pores of the BCPs, and finally replacing the BCPs. At 2 weeks postoperatively, there was a significant difference in bone formation between the 5 groups, including in BV/TV, BMD and Tb.N values, which were the highest in antimiR group, and lowest in the miR group $(\mathrm{P}<0.05)$; this difference between the groups was retained at 4 and 8 weeks postoperatively $(\mathrm{P}<0.05)$. These data suggested that the antimiR group had the most newly formed bone and the highest mineralization rate, while the miR group had the opposite outcome. There was a time-dependent increase in BV/TV, BMD and Tb.Th, and a time-dependent decrease in Tb.N $(\mathrm{P}<0.05)$. For Tb.Th, at 2 weeks the antimiR group was the lowest $(\mathrm{P}<0.01)$, however, at 4 weeks the antimiR group was the highest $(\mathrm{P}<0.01)$ and the miR group was the lowest $(\mathrm{P}<0.05)$ among the five groups; no significant differences were found at 8 weeks postoperatively.

Evaluation of the relative quantity of residual BCP scaffolds in the ROI showed a gradual resorption of BCPs with time. At each time point, the antimiR group had the lowest amount of residual BCP, while the miR group had the most $(\mathrm{P}<0.01)$, which was the opposite to the results observed for newly formed bone. 


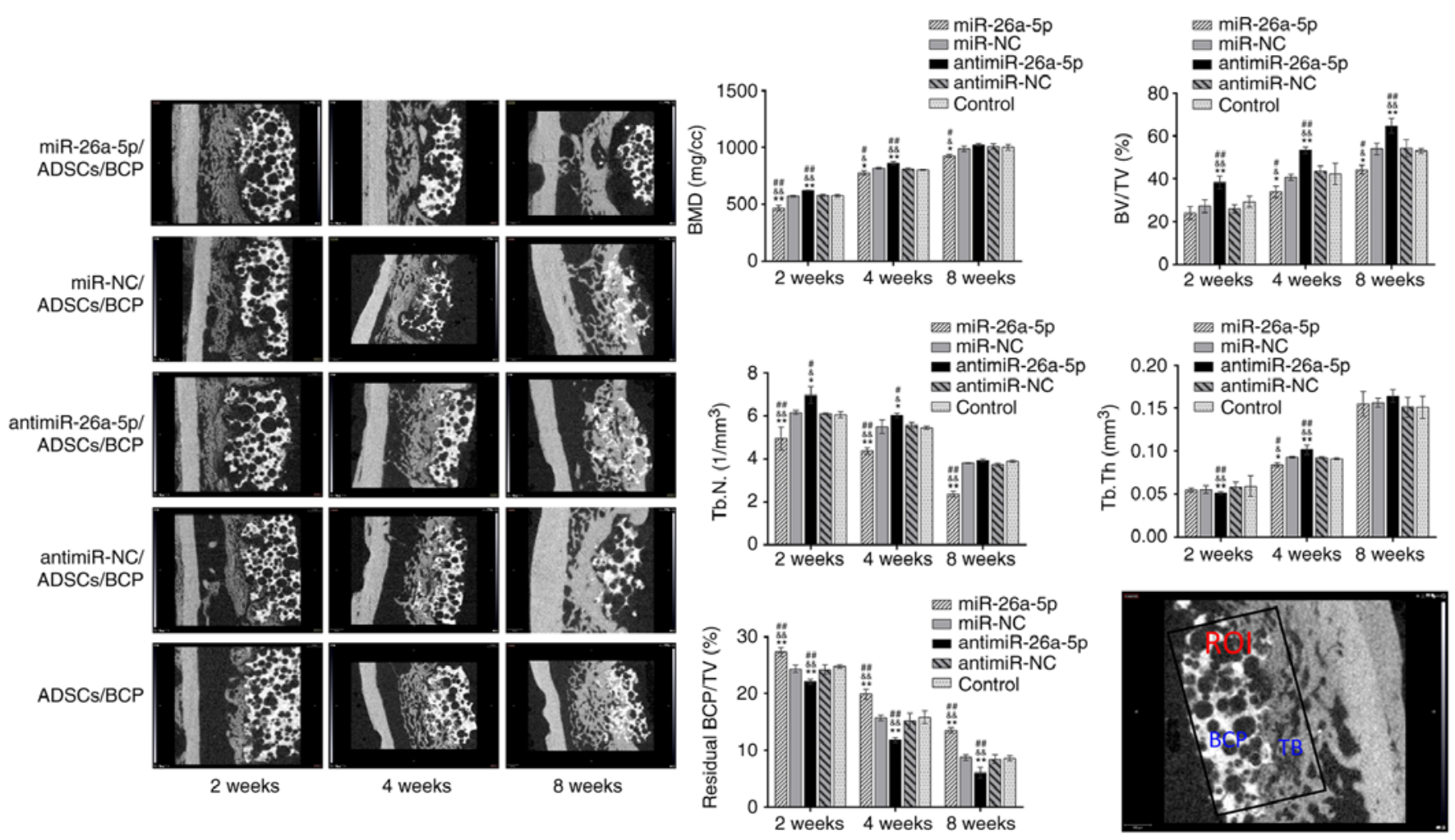

Figure 4. Micro-computed tomography evaluation of bone regeneration and general trabecular microstructure parameters of ROI in the miR, miR-NC, antimiR, antimiR-NC and Control groups. The BMD, BV/TV and Tb.N were significantly lower in the miR group and higher in the antimiR group compared with the other three groups. There was no regular change trend in the pattern of Tb.Th between the five groups at 8 weeks. The percentage of residual BCP was the lowest in the antimiR group, and the greatest in the miR group when comparing the five groups. ${ }^{*} \mathrm{P}<0.05$ and ${ }^{* *} \mathrm{P}<0.01 \mathrm{vs}$. miR-NC; ${ }^{*} \mathrm{P}<0.05$ and ${ }^{\&}{ }^{\&} \mathrm{P}<0.01$ vs. antimiR-NC; ${ }^{\#} \mathrm{P}<0.05$ and ${ }^{\# \#} \mathrm{P}<0.01$ vs. control. ROI, region of interest; miR, microRNA; NC, negative control; ADSCs, adipose-derived mesenchymal stem cells; BCP, biphasic calcium phosphate; W, weeks; BV/TV, ratio of bone volume to total volume; BMD, bone mineral density; Tb.N, trabecular number; Tb.Th, trabecular thickness; TB, tissue-engineered bone.

Histological evaluation of bone regeneration. Representative sections of the 5 groups $(n>3)$ were captured using software coupled with a light microscope. The red color indicates bone and collagen fibers, and the BCPs formed blanks filling the entire defect area (Fig. 5). At 2 weeks postoperatively, there was a thin layer of newly formed bone and collagen fibers between the BCPs and the adjacent native bones and medullar cavities. Additionally, there was a large amount of spot-like collagen fibers, and even new bone in the center, invading into the macro-pores of the BCPs in the antimiR group. Due to the shape of the pores in the BCP scaffolds, the newly formed tissue in the pores manifested as round spots and the blank spaces surrounding them were occupied by residual BCP. There was no significant tissue formation in the pores of the scaffolds in the other 4 groups. At 4 weeks postoperatively, regenerated trabecular bone grew thicker and more abundant compared with at 2 weeks, and had begun mineralization. The macro-pores of the BCPs began to be filled with newly formed bone and collagen fibers in all groups except the miR group. In the antimiR group, the majority of the BCP pores had been filled by woven bones or collagen fibers. BCP degradation could be identified based on the decrease in blank spaces filling the defects. At 8 weeks postoperatively, with the absorption of scaffolds, the spot-like newly formed bone began to merge together in all groups. The defects were already filled with newly formed woven bones, some of which had become mature lamellar bones, and the rebuilding process was performed with the medullary cavity starting to form in the defect area of the miR-NC, antimiR and antimiR-NC groups. The newly formed bones in the miR group did not completely repair the defect without significant medullary cavity formation. In addition, reticulation structures from the $\mathrm{H} \& \mathrm{E}$ sections could be easily identified in the blank area, namely in the residual BCP scaffolds. This was especially true for the antimiR group at both 4 and 8 postoperative weeks, where reticulation structures could be easily identified in the blank area, namely in the residual BCP scaffolds.

Semi-quantitative analysis of the newly formed bone in the defect area corresponded with the results of micro-CT analysis (Fig. 6). Comparison of the five groups revealed that at each time point, the quantity of new bone was the greatest in the antimiR group $(\mathrm{P}<0.01)$ and the lowest in the miR group $(\mathrm{P}<0.05)$.

IHC evaluation of bone regeneration. IHC staining demonstrated that osteoblasts from all 5 groups stained positive for 3 osteogenic-related proteins (RUNX2, OCN and COLI) and two pathway proteins (WNT5A and CaMKII) at 2,4 and 8 weeks postoperatively. The histograms revealed that the time courses of expression were similar, with the highest expression at 2 weeks postoperatively, and a decreasing trend over time (Figs. 7-9). The role of miR-26a-5p and antimiR-26a-5p in new bone regeneration was investigated by comparing the relative expression levels of RUNX2, OCN and COLI in 


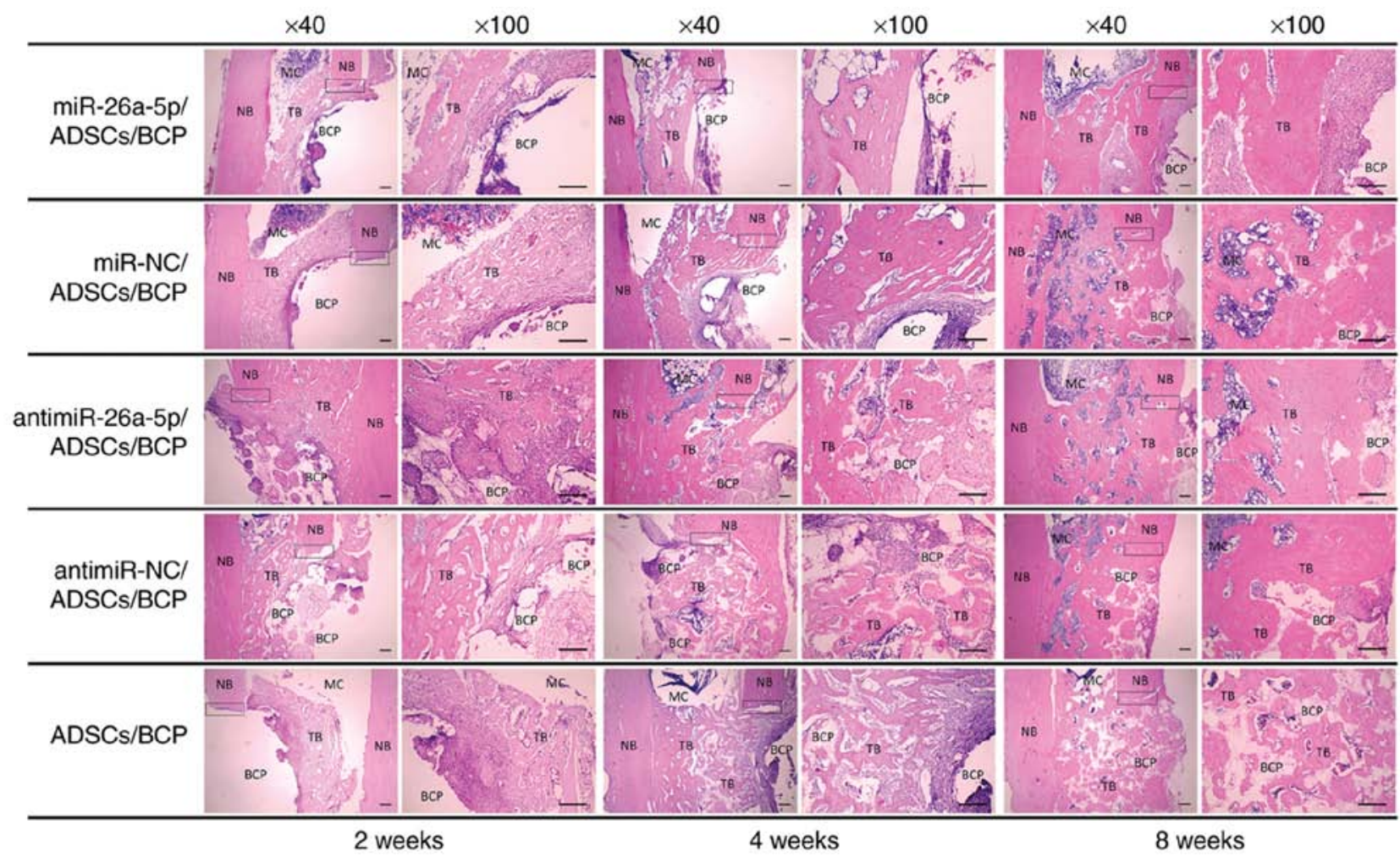

Figure 5. Histological analysis of the regenerated bone in the miR, miR-NC, antimiR, antimiR-NC and Control groups at 2,4 and 8 weeks postoperatively (hematoxylin and eosin staining; magnification, $\mathrm{x} 40$ and x100 as indicated; Scale bars, $200 \mu \mathrm{m}$ ). Squares indicate the connection between the NB and TB. miR, microRNA; NC, negative control; ADSCs, adipose-derived mesenchymal stem cells; BCP, biphasic calcium phosphate; MC, medullar cavity; NB, natural bone; TB, tissue-engineered bone.

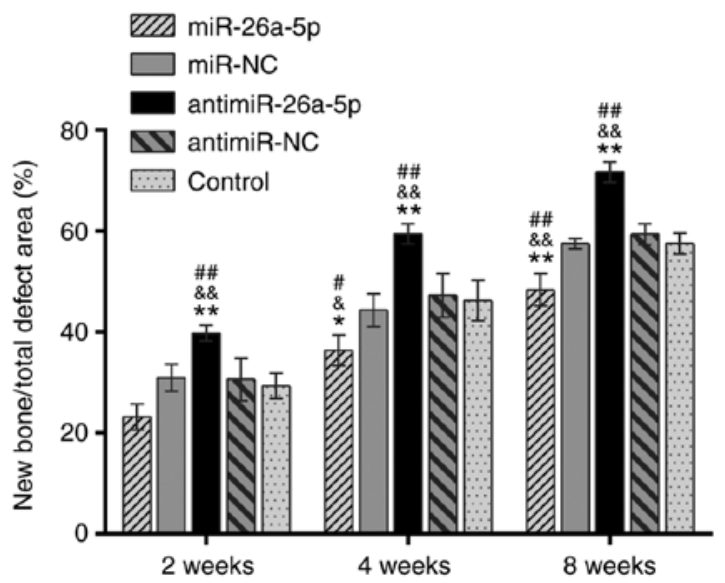

Figure 6. Relative volume of new bone in the total defect area as determined by hematoxylin and eosin evaluation. ${ }^{*} \mathrm{P}<0.05$ and ${ }^{* *} \mathrm{P}<0.01$ vs. miR-NC; ${ }^{\&} \mathrm{P}<0.05$ and ${ }^{\& \&} \mathrm{P}<0.01$ vs. antimiR-NC; ${ }^{*} \mathrm{P}<0.05$ and ${ }^{\# \#} \mathrm{P}<0.01$ vs. control. miR, microRNA; NC, negative control.

the miR and antimiR groups with those in the other groups. Evaluating the proportion of RUNX2-positive cells at $x 400$ magnification revealed that the miR group had a significantly lower proportion of RUNX2-postive osteoblasts compared with the miR-NC group $(\mathrm{P}<0.05)$, while the antimiR group had a significantly higher number of RUNX2-positive cells compared with the antimiR-NC group $(\mathrm{P}<0.05$; Fig. 7). There was no significant difference between the miR-NC, antimiR-NC and Control groups. The expression of OCN and COLI was similar to that ofRUNX2 (Fig. 8). These data indicated that modified ADSCs seeded onto the BCP scaffolds participated in new bone formation. Furthermore, the expression of WNT5A- and CaMKII-positive cells was increased in the antimiR group and decreased in the miR group at all time points $(\mathrm{P}<0.05$; Fig. 9). Taken together, the results indicated that miR-26a-5p regulated in vivo bone formation by ADSCs by inhibiting WNT5A and CaMKII expression.

\section{Discussion}

The primary aim of the present study was to construct a novel and effective TEBG using antimiR-26a-5p-modified ADSCs combined with a BCP scaffold. The cell-material complexes were initially constructed successfully in vitro. BCPs were previously shown to have a good ability for cell attachment and proliferation $(32,33)$, and this was consistent with the SEM and CCK8 assay results of the present study. The SEM results demonstrated that the ADSCs adhered and grew on the BCP scaffolds after 7 days of co-culture, with a fusiform or polygon morphology. The results of the CCK- 8 assay indicated that the proliferation of ADSCs on the BCPs of the 5 groups was not different, which meant that the cell vitality was satisfactory in the 5 groups. These cell-scaffold complexes could be implanted to repair bone defects.

The present study investigated the role of miR-26a-5p and antimiR-26a-5p on bone regeneration in a rat femoral defect model. Quantitative analysis of micro-CT showed that the quantity and quality of newly formed bone were the lowest in the miR group and highest in the antimiR group when compared with the miR-NC, antimiR-NC and Control groups 

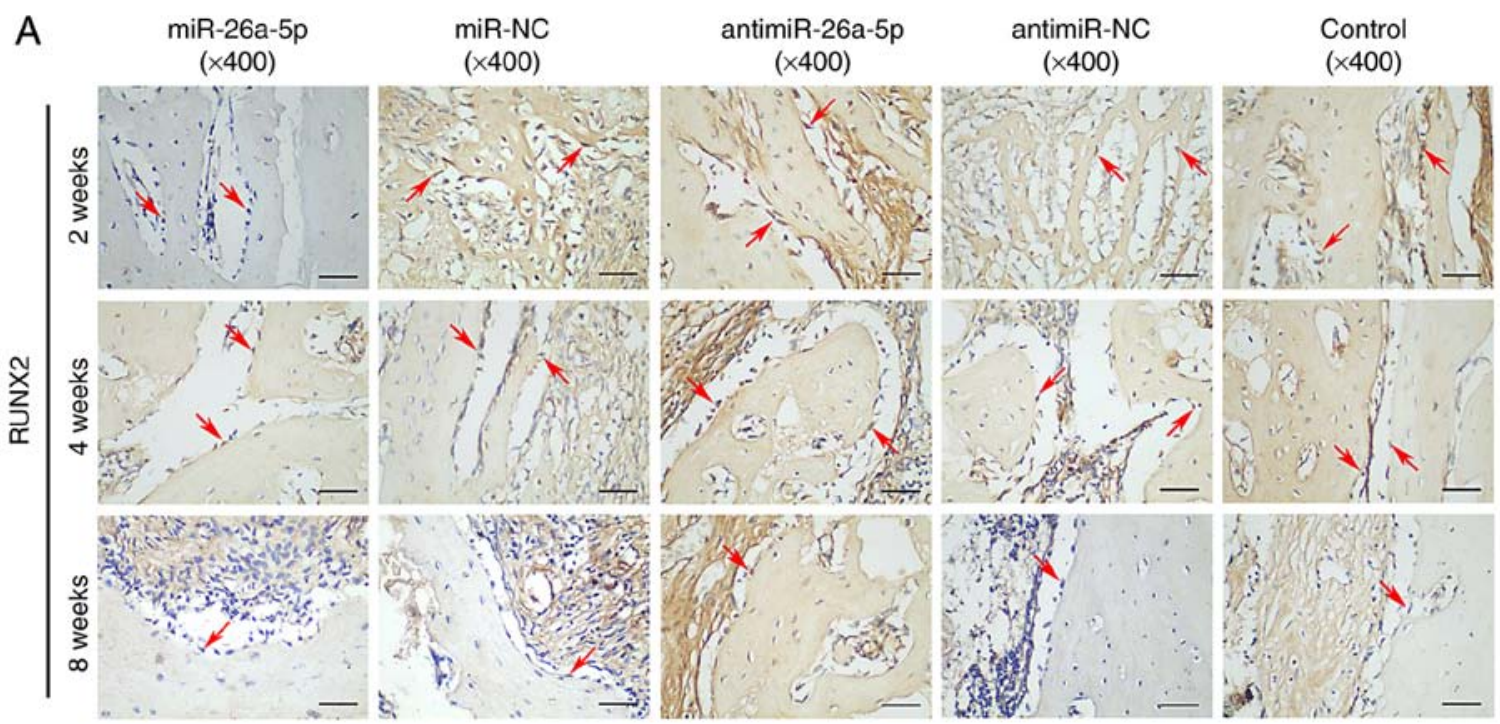

B
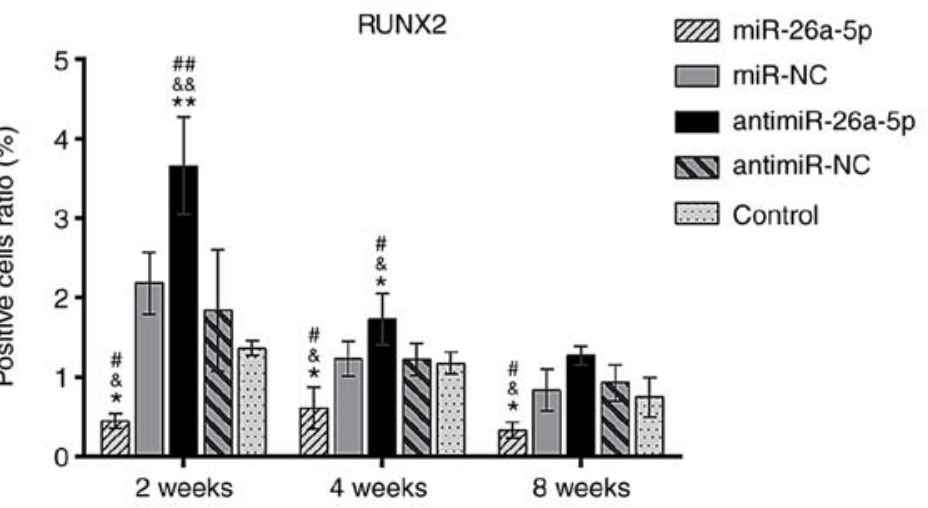

Figure 7. Immunohistochemical analysis specific for RUNX2 in the miR, miR-NC, antimiR, antimiR-NC and Control groups at 2,4 and 8 weeks, postoperatively. (A) The cytoplasm of RUNX2-positive osteoblasts stained brown along the surface of new bone (magnification, $\mathrm{x} 400$; scale bars, $50 \mu \mathrm{m}$ ). (B) Relative area of RUNX2-positive osteoblasts to the total area of the images. Red arrows indicate the positively stained osteoblasts. ${ }^{*} \mathrm{P}<0.05$ and ${ }^{* *} \mathrm{P}<0.01 \mathrm{vs}$. miR-NC; ${ }^{\&} \mathrm{P}<0.05$ and ${ }^{\& \&} \mathrm{P}<0.01$ vs. antimiR-NC; ${ }^{*} \mathrm{P}<0.05$ and ${ }^{\# \#} \mathrm{P}<0.01$ vs. control. miR, microRNA; NC, negative control; RUNX2, Runt-related transcription factor 2.

based on the BMD, BV/TV and Tb.N data $(\mathrm{P}<0.05)$. H\&E sections from the 5 groups indicated that the amount of newly generated bone was also the highest in the antimiR group and the lowest in the miR group $(\mathrm{P}<0.05)$. In addition, the relative expressions of RUNX2, OCN and COLI proteins were the lowest in the miR group and the highest in the antimiR group compared with the other 3 groups $(\mathrm{P}<0.05)$. There were no significant differences in the expression of these proteins between the miR-NC, antimiR-NC and Control groups. Taken together, the results from the gross observations, X-ray and micro-CT evaluations, and $\mathrm{H} \& \mathrm{E}$ and IHC examinations all suggested that miR-26a-5p-modified ADSCs combined with BCP scaffolds attenuated the bone formation process. By contrast, antimiR-26a-5p-modified ADSCs combined with BCP scaffolds promoted the process compared with the unmodified ADSCs. Combined with the results of our previous study (29), the present data suggested that miR-26a-5p functions as a negative regulator of the osteogenic differentiation of ADSCs and osteogenesis, while antimiR-26a-5p effectively promotes osteogenic differentiation and osteogenesis. antimiR-26a-5p-modified ADSCs combined with BCP scaffolds should therefore be able to promote bone regeneration effectively, providing potential for bone repair and reconstruction in the load-bearing area.
Although a number of studies have investigated techniques to promote MSC osteogenic differentiation through miRNA modifications (34-36), the molecular mechanisms underlying osteogenesis are not completely understood. We previously showed that miR-26a-5p inhibited the translation of Wnt5a by directly binding to the 3'-UTR of Wnt5a (29). In the present study, IHC staining was conducted to analyze the expression of WNT5A in osteoblasts. The results showed that the ratio of WNT5a-positive cells to negative cells was the lowest in the miR group and the highest in the antimiR group compared with the other groups $(\mathrm{P}<0.05)$, suggesting that miR-26a-5p negatively regulated the expression of WNT5A and antimiR-26a-5p promoted the expression of WNT5A. WNT5A has been identified by many studies as a noncanonical Wnt ligand and activates two noncanonical Wnt pathways, one of which is the $\mathrm{Wnt} / \mathrm{Ca}^{2+}$ signaling pathway $(37,38)$. Additionally, our previous study proved that miR-26a-5p inhibits the osteogenic differentiation of ADSCs by downregulating the Wnt5a/Ca ${ }^{2+}$ signaling pathway (29). Therefore, the present study used IHC staining to demonstrate that the expression of CaMKII in $\mathrm{Wnt} / \mathrm{Ca}^{2+}$, a downstream mediator of WNT5A via the $\mathrm{Wnt} / \mathrm{Ca}^{2+}$ pathway (29), exhibited a similar pattern to WNT5A. Taken together, the results indicated that miR-26a-5p inhibited the expression of WNT5A, leading to low expression of 
A
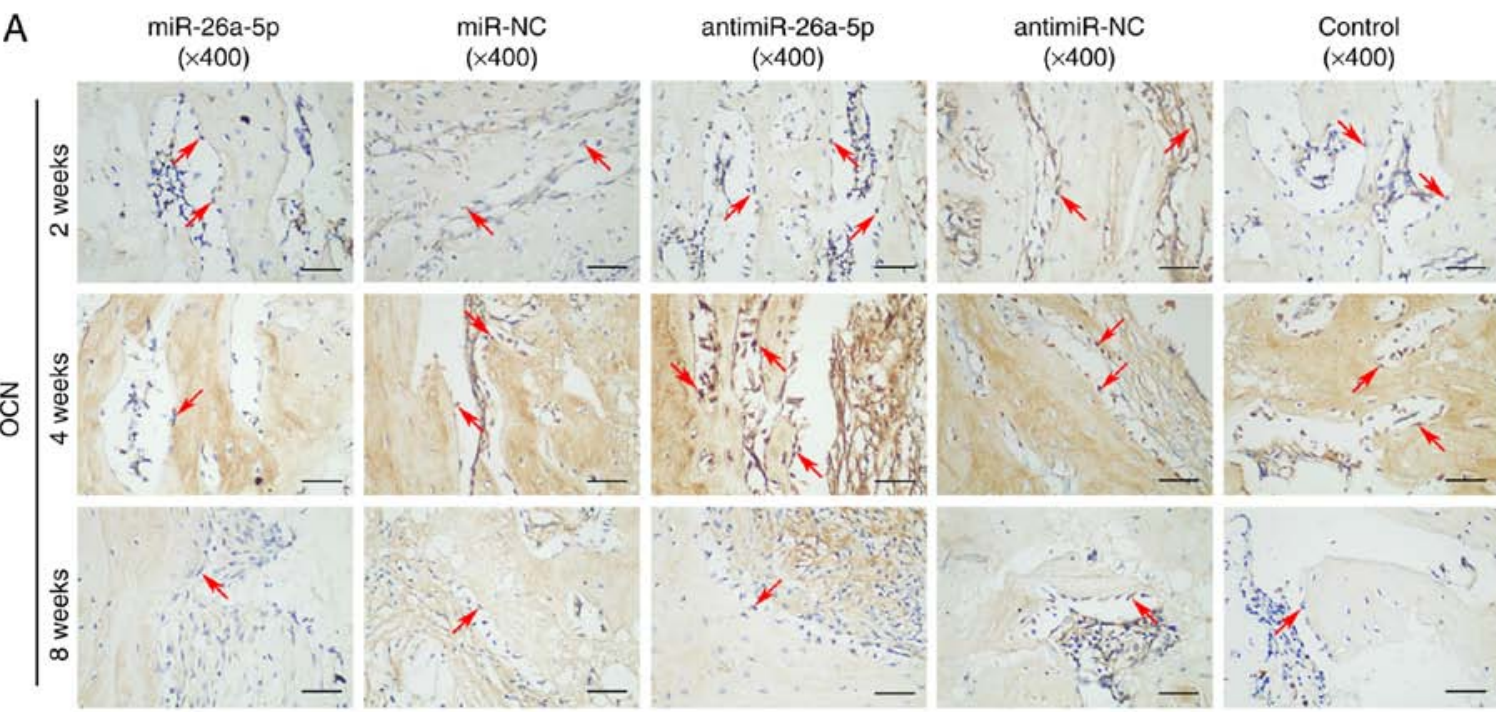

B
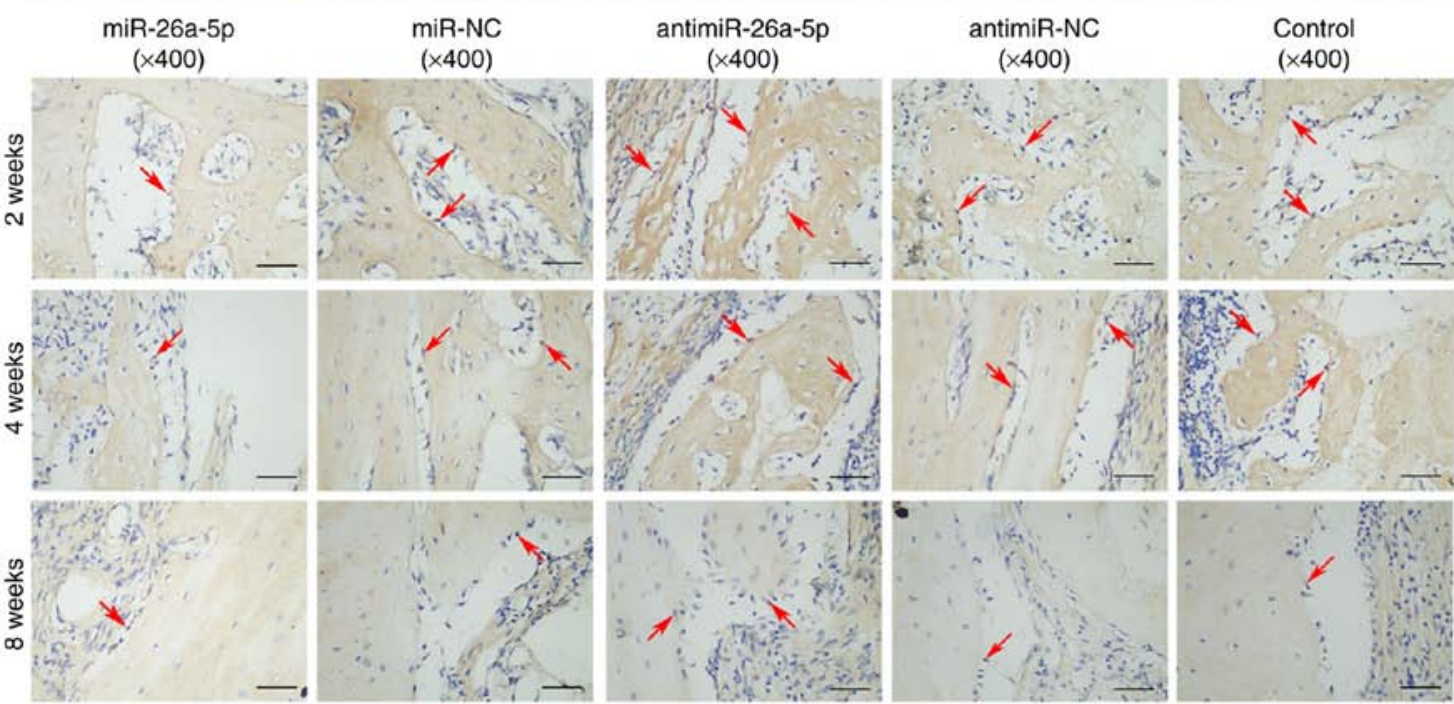

C

$\mathrm{CCN}$

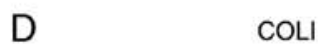

COLI

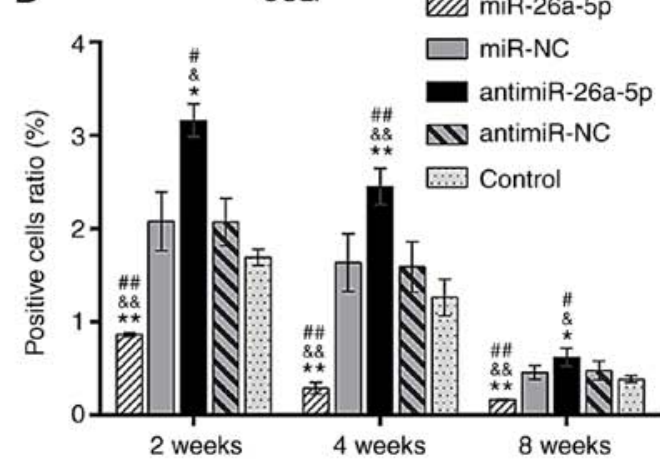

Figure 8. Immunohistochemical analysis specific for OCN and COLI in the miR, miR-NC, antimiR, antimiR-NC and Control groups at 2,4 and 8 weeks, postoperatively. (A) The cytoplasm of OCN-positive osteoblasts was stained brown (magnification, x400; scale bars, $50 \mu \mathrm{m}$ ). (B) COLI-positive osteoblasts were stained brown along the surface of new bones (magnification, $\mathrm{x} 400$; scale bars, $50 \mu \mathrm{m}$ ). (C) Relative area of OCN-positive osteoblasts to the total area of images. (D) Relative area of COLI-positive osteoblasts to the total area of images. Red arrows indicated the positively-stained osteoblasts. "P<0.05 and ${ }^{* *} \mathrm{P}<0.01$ vs. miR-NC; ${ }^{\&} \mathrm{P}<0.05$ and ${ }^{\& \&} \mathrm{P}<0.01$ vs. antimiR-NC; ${ }^{*} \mathrm{P}<0.05$ and ${ }^{\# \#} \mathrm{P}<0.01$ vs. control. miR, microRNA; NC, negative control; OCN, osteocalcin; COLI, collagen I.

CaMKII, finally resulting in attenuation of bone regeneration. The present study demonstrated that the $\mathrm{Wnt} / \mathrm{Ca}^{2+}$ signaling pathway regulated osteogenesis by miR-26a-5p, and these results were consistent with the conclusions from our previous study (29).
The ideal properties for scaffolds of tissue-engineering bone include optimal biocompatibility, biodegradability, osteoconduction, osteoinduction, plasticity, mechanical strength, and the ability to be easily sterilized and preserved $(11,39)$. BCPs are composed of calcium phosphate, the same inorganic material 
A
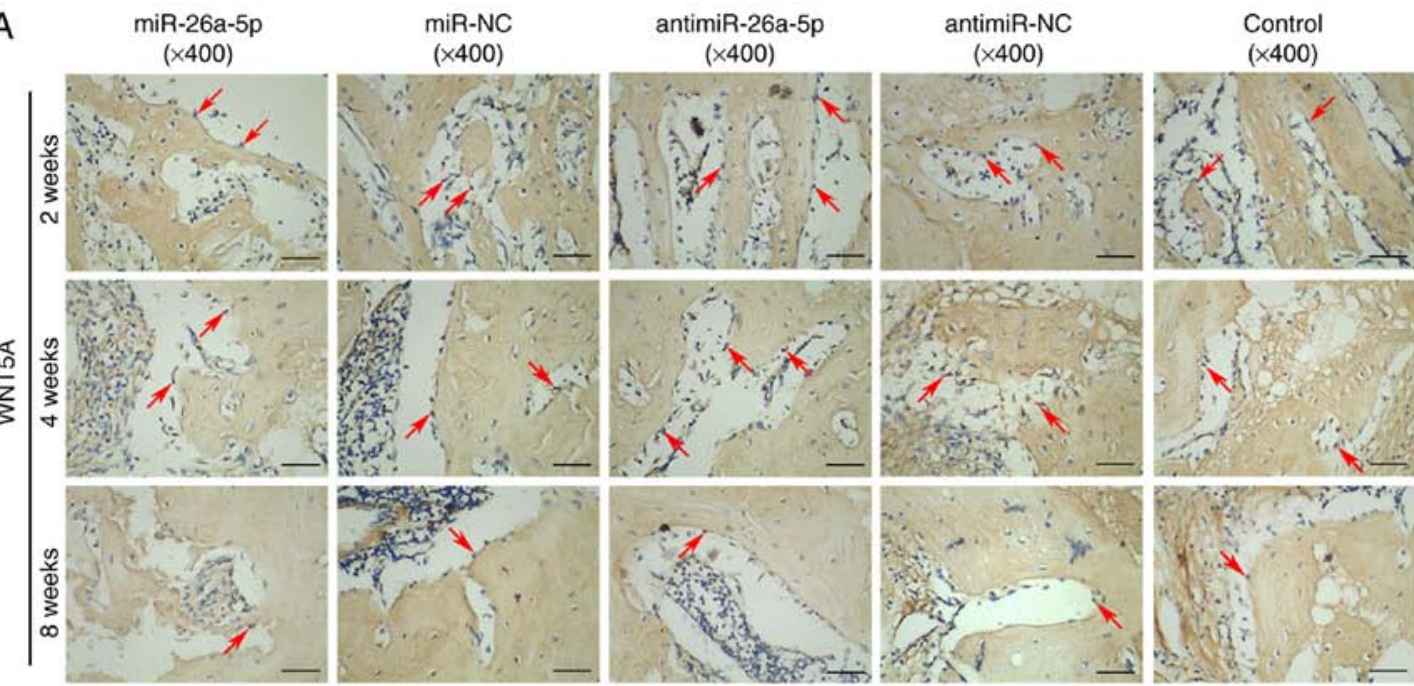

B
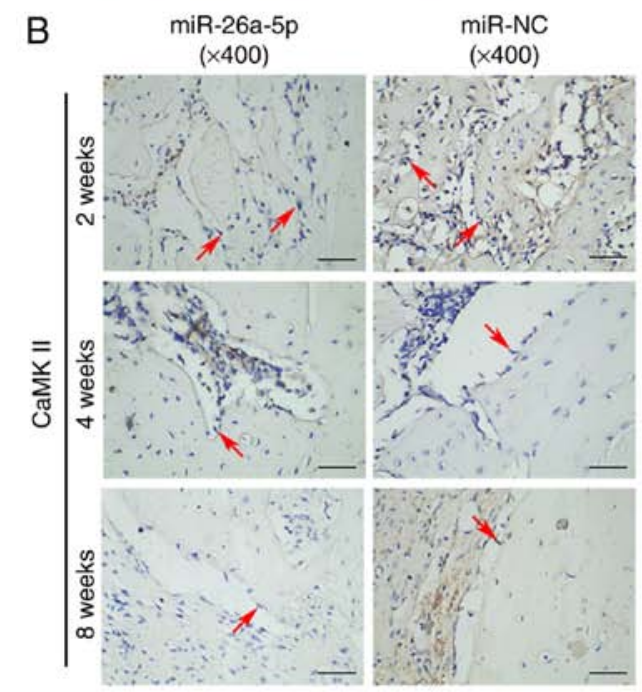

antimiR-26a-5p

antimiR-NC

Control

$(\times 400)$

$(\times 400)$

$(\times 400)$
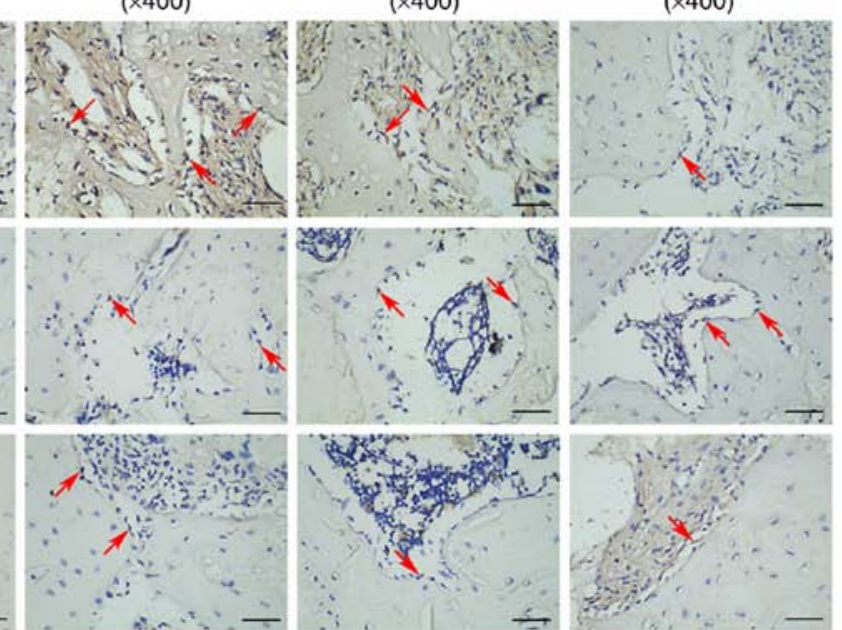

C

WNT5A
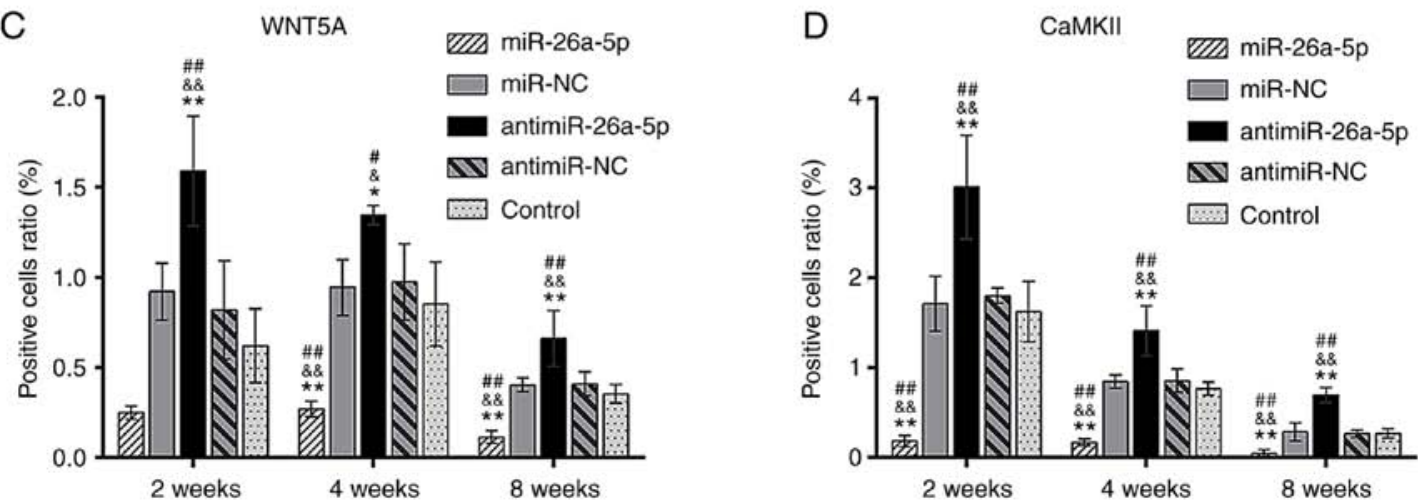

Figure 9. Immunohistochemical analysis specific for WNT5A and CaMKII in the miR, miR-NC, antimiR, antimiR-NC and Control groups at 2,4 and 8 weeks, postoperatively. (A) Immunohistochemical staining specific for WNT5A. The cytoplasm of WNT5A-positive osteoblasts was stained brown (magnification, $\mathrm{x} 400$; scale bars, $50 \mu \mathrm{m}$ ). (B) Immunohistochemical staining specific for CaMKII. The cytoplasm of CaMKII-positive osteoblasts was stained brown or yellow (magnification, x400; scale bars, $50 \mu \mathrm{m}$ ). (C) Relative area of WNT5A-positive osteoblasts to the total area of images. (D) Relative area of CaMKII-positive osteoblasts to the total area of images. Red arrows indicate the positively stained osteoblasts. ${ }^{*} \mathrm{P}<0.05$ and ${ }^{* *} \mathrm{P}<0.01$ vs. miR-NC; ${ }^{\text {\& }} \mathrm{P}<0.05$ and ${ }^{\& \&} \mathrm{P}<0.01$ vs. antimiR-NC; ${ }^{\#} \mathrm{P}<0.05$ and ${ }^{\# \#} \mathrm{P}<0.01$ vs. control. miR, microRNA; NC, negative control; CaMKII, calmodulin-dependent protein kinase II; WNT5A, Wnt family member 5A.

which comprises the natural skeleton $(12,13,15)$. The present study suggested that tissue-engineered bone constructed with BCP scaffolds could successfully repair the bone defect, and $\mathrm{BCP}$ could be absorbed in vivo $(40,41)$. These results validated earlier studies that reported that BCP is the ideal choice of scaffolds for tissue-engineered bone $(12,13,15)$. HA, with a calcium to phosphorus ratio $(\mathrm{Ca} / \mathrm{P})$ of 1.67 , is considered to be the stable phase and is hardly absorbed, which may be an obstacle for its use in bone regeneration $(42,43)$. By contrast, $\beta-\mathrm{TCP}$, with a $\mathrm{Ca} / \mathrm{P}$ ratio of 1.5 , was easily absorbed (43). BCPs 
are combinations of HA and $\beta$-TCP, and have the advantages of both; the combinations compensate for the individual disadvantages of each material $(44,45)$. The biological properties of BCPs, including the biocompatibility, bioactivity, biodegradation, osteoconduction, osteoinduction and cell adhesion are influenced by the physicochemical properties, the most important of which is the HA/ $\beta$-TCP ratio $(39,46,47)$. Porous BCPs with a lower HA/ $\beta$-TCP ratio have been shown to have better degradation and osteoinduction, which correlates with better osteogenesis (48). By contrast, a high ratio of $\beta$-TCP was shown to correlate with poor mechanical strength and a rapid degradation rate, leading to cracks between BCPs and the newly formed bone, and failure to regenerate bone (49). Previous results revealed that a porous BCP with a HA/ $\beta$-TCP ratio of 20/80 had good biocompatibility, bioactivity and a fast degradation rate $(18,19)$. The micro-CT and histological analysis in the present study showed that the residual BCP particles were packed by newly formed bone without cracks in the antimiR group, indicating that the rate of bone formation could compensate for the rate of BCP degradation. In the miR group, however, the residual BCP scaffold was repelled, resulting in no support for further bone formation to fill the defect. These results suggested that an increased bone formation rate may lead to successful bone reconstruction with $\mathrm{BCP}$ scaffolds, covering the shortages of BCP20/80.

Additionally, micro-CT was used to evaluate the degradation rates of $\mathrm{BCP}$ scaffolds in the 5 groups. The present results revealed an accelerated degradation rate in the antimiR group, and decelerated the degradation rate in the miR group compared with the miR-NC, antimiR-NC and Control groups. Simultaneously, the antimiR group exhibited the fastest bone formation rate while the miR group had the slowest bone formation rate. These data suggested that the differences in $\mathrm{BCP}$ degradation rates may be related to the application of miR-26a-5p and antimiR-26a-5p. It was previously reported that the microenvironment of bone formation and extracellular matrix facilitated osteoclast adhesion, which is important for material degradation (50). In addition, osteoblasts provide a microenvironment for osteoclastogenesis, and WNT5A secreted by osteoblasts is a new co-stimulatory cytokine for osteoclastogenesis; furthermore, receptor tyrosine kinase-like orphan receptor 2 acts as the functional receptor of Wnt5a in osteoclast precursors (51). Therefore, increased expression of osteoblasts and WNT5A in the antimiR group activated the function of osteoclasts and accelerated the degradation of $\mathrm{BCP}$, a process which requires further investigation.

There were 3 key osteogenic-related proteins that had similar expression profiles among the 5 groups during the time course of the present study. Since OCN, COLI and RUNX2 are markers of osteoblasts and are mainly secreted by them, the ratio of positive cells represents the quantity of osteoblasts and their viability, which further indicates better osteogenesis in vivo. Combined with the results of $\mathrm{H} \& \mathrm{E}$ and micro-CT, IHC analysis may prove the regulatory function of miR-26a-5p in bone formation at the molecular level. The expression of RUNX2, OCN and COLI reached a peak at 2 postoperative weeks, and had slowly declined by 4 postoperative weeks; they reached very low levels at 8 postoperative weeks. A previous study reported the same expression pattern of OCN and RUNX2 as the present study, and that OCN and RUNX2 reached a peak at an early stage (52). However, OCN and COLI are considered to be the late-stage osteogenic markers (53-55). Since the present data indicated that miR-26a-5p and antimiR-26a-5p had the opposite effects on osteogenesis in the miR and antimiR groups, we hypothesized that the early-stage high expression of OCN and COLI may be associated with the calcium and phosphate ions released into the microenvironment by the BCP scaffolds. Previous studies have reported that, with a degradation rate determined by the composition of BCPs, suitable concentrations of ions are released into the microenvironment of tissue-engineered bone, triggering an early and high expression of osteogenic-related genes and proteins through the BMP signaling pathway (56). Elevated $\mathrm{Ca}^{2+}$ concentrations were also shown to promote the recruitment of bone marrow progenitor cells in vivo, regulate the expression of bone morphogenetic protein and COLI in osteoblasts, and modulate the osteoinduction of MSCs $(57,58)$. It is therefore possible that the rapid degradation of BCP20/80 may lead to increased extracellular $\mathrm{Ca}^{2+}$ concentrations, resulting in the early-stage elevated expression of OCN and COLI in osteoblasts. Further investigations are necessary to examine the concentration of ions in the extracellular matrix to verify this hypothesis.

In conclusion, the results of the present study revealed that miR-26a-5p was a negative regulator of osteogenic differentiation of ADSCs. miR-26a-5p-modified ADSCs combined with BCP scaffolds significantly attenuated bone regeneration, while antimiR-26a-5p-modified ADSCs enhanced bone formation. Based on the variation in the expression of WNT5A and its downstream protein, CaMKII, as well as that of osteogenesis-related proteins, it is likely that miR-26a-5p regulated the osteogenic differentiation of ADSCs and bone formation via the $\mathrm{Wnt} / \mathrm{Ca}^{2+}$ signaling pathway. The present results indicated a potential regulatory role for miR-26a-5p in bone regeneration. It was also suggested that the combination of antimiR-26a-5p-modified ADSCs and the BCP scaffold could provide a promising and effective strategy for bone repair and reconstruction. The present results provide important preclinical data to support the application of miR-26a-5p in bone-related diseases.

\section{Acknowledgements}

Not applicable.

\section{Funding}

The present study was supported by grants from the National Nature Science Foundation of China (grant nos. 31570950, 10502037 and 31070833) and the Science and Technology Foundation of Sichuan Province (grant. nos. 2017SZ0032, 2010GZ0225, 2011GZ0335 and 2009SZ0139).

\section{Availability of data and materials}

All data generated or analyzed during this study are included in this published article.

\section{Authors' contributions}

XY conceived and designed the study, collected, analyzed and interpreted the data, and wrote the manuscript. LH conceived 
and designed the study, and collection and presented the data. HL analyzed and interpreted the data, and wrote the manuscript. ZG analyzed and interpreted the data. YH conceived and designed the study, and collected the data. SL performed the statistical analysis. TL was involved in the animal experiments. WTa was involved in cell culture and gene transfection. WTi conceived and designed the study. JL conceived and designed the study, wrote the manuscript, and gave final approval of the manuscript.

\section{Ethics approval and consent to participate}

All experimental procedures involving animals were approved by the Animal Research Committee of Sichuan University.

\section{Patient consent for publication}

Not applicable.

\section{Competing interests}

The authors declare that they have no competing interests.

\section{References}

1. Lee JE, Kim MB, Han DH, Pyo SH and Lee YH: One-barre microsurgical fibula flap for reconstruction of large defects of the femur. Ann Plast Surg 80: 373-378, 2018.

2. Mohseni M,Jahandideh A, Abedi G, Akbarzadeh A and Hesaraki S Assessment of tricalcium phosphate/collagen (TCP/collagene) nanocomposite scaffold compared with hydroxyapatite (HA) on healing of segmental femur bone defect in rabbits. Artif Cells Nanomed Biotechnol 46: 242-249, 2018.

3. Sakkas A, Schramm A, Winter K and Wilde F: Risk factors for post-operative complications after procedures for autologous bone augmentation from different donor sites. J Craniomaxillofac Surg 46: 312-322, 2018

4. Burk T, Del Valle J, Finn RA and Phillips C: Maximum quantity of bone available for harvest from the anterior iliac crest posterior iliac crest, and proximal tibia using a standardized surgical approach: A cadaveric study. J Oral Maxillofac Surg 74: 2532-2548, 2016.

5. Aponte-Tinao LA, Albergo JI, Ayerza MA, Muscolo DL, Ing FM and Farfalli GL: What Are the complications of allograft reconstructions for sarcoma resection in children younger than 10 years at long-term followup? Clin Orthop Relat Res 476: 548-555, 2018

6. Khodakaram-Tafti A, Mehrabani D, Shaterzadeh-Yazdi H, Zamiri B and Omidi M: Tissue engineering in maxillary bone defects. World J Plast Surg 7: 3-11, 2018.

7. Diomede F, Gugliandolo A, Cardelli P, Merciaro I, Ettorre V, Traini T, Bedini R, Scionti D, Bramanti A, Nanci A, et al Three-dimensional printed PLA scaffold and human gingival stem cell-derived extracellular vesicles: A new tool for bone defect repair. Stem Cell Res Ther 9: 104, 2018.

8. Reichert JC, Saifzadeh S, Wullschleger ME, Epari DR, Schütz MA, Duda GN, Schell H, van Griensven M, Redl H and Hutmacher DW: The challenge of establishing preclinical models for segmental bone defect research. Biomaterials 30: 2149-2163, 2009.

9. Le BQ, Nurcombe V, Cool SM, van Blitterswijk CA, de Boer J and LaPointe VLS: The components of bone and what they can teach us about regeneration. Materials (Basel) 11: E14, 2017.

10. Horch RE, Beier JP, Kneser U and Arkudas A: Successful human long-term application of in situ bone tissue engineering. J Cell Mol Med 18: 1478-1485, 2014.

11. Pennesi G, Scaglione S, Giannoni P and Quarto R: Regulatory influence of scaffolds on cell behavior: How cells decode biomaterials. Curr Pharm Biotechnol 12: 151-159, 2011.

12. Uzeda MJ, de Brito Resende RF, Sartoretto SC, Alves ATNN, Granjeiro JM and Calasans-Maia MD: Randomized clinical trial for the biological evaluation of two nanostructured biphasic calcium phosphate biomaterials as a bone substitute. Clin Implant Dent Relat Res 19: 802-811, 2017.
13. Yun PY, Kim YK, Jeong KI, Park JC and Choi YJ: Influence of bone morphogenetic protein and proportion of hydroxyapatite on new bone formation in biphasic calcium phosphate graft: Two pilot studies in animal bony defect model. J Craniomaxillofac Surg 42: 1909-1917, 2014.

14. Wang S, Zhang Z, Zhao J, Zhang X, Sun X, Xia L, Chang Q, Ye D and Jiang X: Vertical alveolar ridge augmentation with beta-tricalcium phosphate and autologous osteoblasts in canine mandible. Biomaterials 30: 2489-2498, 2009.

15. Shuang Y, Yizhen L, Zhang Y, Fujioka-Kobayashi M, Sculean A and Miron RJ: In vitro characterization of an osteoinductive biphasic calcium phosphate in combination with recombinant BMP2. BMC Oral Health 17: 35, 2016.

16. Dragonas P, Palin C, Khan S, Gajendrareddy PK and Weiner WD: Complications associated with the use of recombinant human bone morphogenic protein-2 in ridge augmentation: A case report. J Oral Implantol 43: 351-359, 2017.

17. Uludag H, D'Augusta D, Palmer R, Timony G and Wozney J: Characterization of rhBMP-2 pharmacokinetics implanted with biomaterial carriers in the rat ectopic model. J Biomed Mater Res 46: 193-202, 1999.

18. Arinzeh TL, Tran T, Mcalary J and Daculsi G: A comparative study of biphasic calcium phosphate ceramics for human mesenchymal stem-cell-induced bone formation. Biomaterials 26 : 3631-3638, 2005.

19. van Esterik FA, Zandieh-Doulabi B, Kleverlaan CJ and Klein-Nulend J: Enhanced Osteogenic and Vasculogenic differentiation potential of human adipose stem cells on biphasic calcium phosphate scaffolds in fibrin gels. Stem Cells Int 2016: 1934270, 2016

20. Lian JB, Stein GS, van Wijnen AJ, Stein JL, Hassan MQ, Gaur T and Zhang Y: MicroRNA control of bone formation and homeostasis. Nat Rev Endocrinol 8: 212-227, 2012.

21. Zuo B, Zhu J, Li J, Wang C, Zhao X, Cai G, Li Z, Peng J, Wang P, Shen C, et al: MicroRNA-103a functions as a mechanosensitive microRNA to inhibit bone formation through targeting Runx2. J Bone Miner Res 30: 330-345, 2015.

22. Hupkes M, Sotoca AM, Hendriks JM, van Zoelen EJ and Dechering KJ: Micro-RNA miR-378 promotes BMP2-induced osteogenic differentiation of mesenchymal progenitor cells BMC Mol Biol 15: 1, 2014.

23. Wang Q, Cai J, Cai XH and Chen L: MiR-346 regulates osteogenic differentiation of human bone marrow-derived mesenchymal stem cells by targeting the $\mathrm{Wnt} / \beta$-catenin pathway. PLoS One 8: e72266, 2013

24. Bartel DP: MicroRNAs: Target recognition and regulatory functions. Cell 136: 215-233, 2009.

25. Li JW, Hu C, Han L, Liu L, Jing W, Tang W, Tian WD and Long J: MiR-154-5p regulates osteogenic differentiation of adipose-derived mesenchymal stem cells under tensile stress through the Wnt/PCP pathway by targeting Wnt11. Bone 78: 130-141, 2015.

26. Wu T, Zhou H, Hong Y, Li J, Jiang X and Huang H: MiR-30 family members negatively regulate osteoblast differentiation. J Biol Chem 287: 7503-7511, 2012.

27. Li Z, Hassan MQ, Volinia S, van Wijnen AJ, Stein JL, Croce CM, Lian JB and Stein GS: A microRNA signature for a BMP2-induced osteoblast lineage commitment program. Proc Natl Acad Sci USA 105: 13906-13911, 2008.

28. Suh JS,Lee JY,Choi YS, Chong PC and Park YJ: Peptide-mediated intracellular delivery of miRNA-29b for osteogenic stem cell differentiation. Biomaterials 34: 4347-4359, 2013.

29. Li S, Hu C, Li J, Liu L, Jing W, Tang W, Tian W and Long J: Effect of miR-26a-5p on the Wnt/Ca(2+) Pathway and Osteogenic differentiation of mouse Adipose-Derived mesenchymal stem cells. Calcif Tissue Int 99: 174-186, 2016.

30. Choudhery MS, Badowski M, Muise A and Harris DT: Comparison of human mesenchymal stem cells derived from adipose and cord tissue. Cytotherapy 15: 330-343, 2013.

31. Ansari S, Diniz IM, Chen C, Sarrion P, Tamayol A, Wu BM and Moshaverinia A: Human periodontal ligament- and gingiva-derived mesenchymal stem cells promote nerve regeneration when encapsulated in alginate/hyaluronic acid 3D scaffold. Adv Healthc Mater: Oct 27, 2017 (Epub ahead of print). Doi: 10.1002/adhm.201700670.

32. Chen Y, Wang J, Zhu XD, Tang ZR, Yang X, Tan YF, Fan YJ and Zhang XD: Enhanced effect of $\beta$-tricalcium phosphate phase on neovascularization of porous calcium phosphate ceramics: In vitro and in vivo evidence. Acta Biomater 11: 435-448, 2015. 
33. Huang L, Zhou B, Wu H, Zheng L and Zhao JM: Effect of apatite formation of Biphasic calcium phosphate (BCP) on the osteoblastogenesis using simulated body fluid with or without bovine serum albumin. Mater Sci Eng C Mater Biol Appl 70: 955-961, 2017.

34. Meng YB, Li X, Li ZY, Zhao J, Yuan XB, Ren Y, Cui ZD, Liu YD and Yang XJ: MicroRNA-21 promotes osteogenic differentiation of mesenchymal stem cells by the $\mathrm{PI} 3 \mathrm{~K} / \beta$-catenin pathway. J Orthop Res 33: 957-964, 2015.

35. Liao YH, Chang YH, Sung LY, Li KC, Yeh CL, Yen TC, Hwang SM, Lin KJ and Hu YC: Osteogenic differentiation of adipose-derived stem cells and calvarial defect repair using baculovirus-mediated co-expression of BMP-2 and miR-148b. Biomaterials 35: 4901-4910, 2014.

36. Huang S, Wang S, Bian C, Yang Z, Zhou H, Zeng Y, Li H, Han Q and Zhao RC: Upregulation of miR-22 promotes osteogenic differentiation and inhibits adipogenic differentiation of human adipose tissue-derived mesenchymal stem cells by repressing HDAC6 protein expression. Stem Cells Dev 21: 2531-2540, 2012

37. Wang Y, Li YP, Paulson C, Shao JZ, Zhang X, Wu M and Chen W: Wnt and the Wnt signaling pathway in bone development and disease. Front Biosci (Landmark Ed) 19: 379-407, 2014.

38. Martineau X, Abed É, Martel-Pelletier J, Pelletier JP and Lajeunesse D: Alteration of Wnt5a expression and of the non-canonical Wnt/PCP and Wnt/PKC-Ca ${ }^{2+}$ pathways in human osteoarthritis osteoblasts. PLoS One 12: e0180711, 2017.

39. Lacroix D, Chateau A, Ginebra MP and Planell JA: Micro-finite element models of bone tissue-engineering scaffolds. Biomaterials 27: 5326-5334, 2006.

40. Bouler JM, Pilet P, Gauthier O and Verron E: Biphasic calcium phosphate ceramics for bone reconstruction: A reviewof biological response. Acta Biomater 53: 1-12, 2017.

41. Tang XH, Mao LX, Liu JQ, Yang Z, Zhang W, Shu MJ, Hu NT, Jiang LY and Fang B: Fabrication, characterization and cellular biocompatibility of porous biphasic calcium phosphate bioceramic scaffolds with different pore sizes. Ceram Int 42: 15311-15318, 2016.

42. Wu Y, Xia L, Zhou Y, Ma W, Zhang N, Chang J, Lin KL, Xu YJ and Jiang XQ: Evaluation of osteogenesis and angiogenesis of icariin loaded on micro/nanohybrid structured hydroxyapatite granules as a local drug delivery system forfemoral defect repair. J Mater Chem B 3: 4871-4883, 2015.

43. Ebrahimi M, Botelho MG and Dorozhkin SV: Biphasic calcium phosphates bioceramics (HA/TCP): Concept, physicochemical properties and the impact of standardization of study protocols in biomaterials research. Mater Sci Eng C Mater Biol Appl 71: 1293-1312, 2017.

44. Jensen SS, Bornstein MM, Dard M, Bosshardt DD and Buser D: Comparative study of biphasic calcium phosphates with different $\mathrm{HA} / \mathrm{TCP}$ ratios in mandibular bone defects. Along-term histomorphometric study in minipigs. J Biomed Mater Res B Appl Biomater 90: 171-181, 2009.

45. Zhu Y, Zhang K, Zhao R, Ye X, Chen X, Xiao Z, Yang X, Zhu X, Zhang K, Fan Y and Zhang X: Bone regeneration with micro/nano hybrid-structured biphasic calcium phosphate bioceramics at segmental bone defect and the induced immunoregulation of MSCs. Biomaterials 147: 133-144, 2017.

46. Ng AM, Tan KK, Phang MY, Aziyati O, Tan GH, Isa MR, Aminuddin BS, Naseem M, Fauziah O and Ruszymah BH: Differential osteogenic activity of osteoprogenitor cells on HA and TCP/HA scaffold of tissue engineered bone. J Biomed Mater Res A 85: 301-312, 2008.
47. Ebrahimian-Hosseinabadi M,Etemadifar M and Ashrafizadeh F: Effects of nano-biphasic calcium phosphate composite on bioactivity and osteoblast cell behavior in tissue engineering applications. J Med Signals Sens 6: 237-242, 2016.

48. Huang J, Ten E, Liu G, Finzen M, Yu W, Lee JS, Saiz E and Tomsia AP: Biocomposites of pHEMA with HA/beta-TCP (60/40) for bone tissue engineering: Swelling, hydrolytic degradation, and in vitro behavior. Polymer (Guildf) 54: 1197-1207, 2013.

49. Daculsi G, Bouler JM and LeGeros RZ: Adaptive crystal formation in normal and pathological calcifications in synthetic calcium phosphate and related biomaterials. Int Rev Cytol 172: 129-191, 1997.

50. Monchau F, Lefevre A, Descamps M, Belquin-myrdycz A, Laffargue $\mathrm{P}$ and Hildebrand HF: In vitro studies of human and rat osteoclast activity on hydroxyapatite, beta-tricalcium phosphate, calcium carbonate. Biomol Eng 19: 143-152, 2002.

51. Maeda K, Kobayashi Y, Udagawa N, Uehara S, Ishihara A, Mizoguchi T, Kikuchi Y, Takada I, Kato S, Kani S, et al: Wnt5a-Ror2 signaling between osteoblast-lineage cells and osteoclast precursors enhances osteoclastogenesis. Nat Med 18: 405-412, 2012.

52. Zhang X, Li Y, Chen YE, Chen J and Ma PX: Cell-free 3D scaffold with two-stage delivery of miRNA-26a to regenerate critical-sized bone defects. Nat Commun 7: 10376, 2016.

53. Sun LY, Wu L, Bao CY, Fu CH, Wang XL, Yao JF, Zhang XD and van Blitterswijk CA: Gene expressions of collagen type I, ALP and BMP-4 in osteo-inductive BCP implants show similar pattern to that of natural healing bones. Mater Sci Eng C 29: 1829-1834, 2009.

54. Wang J, Chen Y, Zhu X, Yuan T, Tan Y, Fan Y and Zhang X: Effect of phase composition on protein adsorption and osteoinduction of porous calcium phosphate ceramics in mice. J Biomed Mater Res A 102: 4234-4243, 2014.

55. Yi T, Jun CM, Kim SJ and Yun JH: Evaluation of in vivo osteogenic potential of bone morphogenetic Protein 2-Overexpressing human periodontal ligament stem cells combined with biphasic calcium phosphate block scaffolds in a Critical-Size bone defect model. Tissue Eng Part A 22: 501-512, 2016.

56. Viti F, Landini M, Mezzelani A, Petecchia L, Milanesi L and Scaglione S: Osteogenic differentiation of MSC through calcium signaling activation: Transcriptomics and functional analysis. PLoS One 11: e0148173, 2016.

57. Tang Z, Tan Y, Ni Y, Wang J, Zhu X, Fan Y, Chen X, Yang X and Zhang $\mathrm{X}$ : Comparison of ectopic bone formation process induced by four calcium phosphate ceramics in mice. Mater Sci Eng C Mater Biol Appl 70: 1000-1010, 2017.

58. González-Vázquez A, Planell JA and Engel E: Extracellular calcium and CaSR drive osteoinduction in mesenchymal stromal cells. Acta Biomater 10: 2824-2833, 2014.

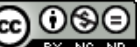

This work is licensed under a Creative Commons Attribution-NonCommercial-NoDerivatives 4.0 International (CC BY-NC-ND 4.0) License. 